\title{
$J$-self-adjoint extensions for second-order linear difference equations with complex coefficients
}

Huaqing Sun ${ }^{1 *}$ and Guojing Ren ${ }^{2}$

"Correspondence:

sunhuaqing_2@163.com

'Department of Mathematics,

Shandong University at Weihai,

Weihai, Shandong 264209, P.R.

China

Full list of author information is

available at the end of the article

\begin{abstract}
This paper is concerned with second-order linear difference equations with complex coefficients which are formally J-symmetric. Both J-self-adjoint subspace extensions and J-self-adjoint operator extensions of the corresponding minimal subspace are completely characterized in terms of boundary conditions.
\end{abstract}

MSC: Primary 39A70; secondary 47A06

Keywords: difference equation; complex coefficient; J-Hermitian subspace; $J$-self-adjoint subspace extension; defect index

\section{Introduction}

In this paper, we consider the following second-order linear difference equation with complex coefficients:

$$
\tau(x)(t):=-\nabla(p(t) \Delta x(t))+q(t) x(t)=\lambda w(t) x(t), \quad t \in I,
$$

where $I$ is the integer set $\{t\}_{t=a}^{b}, a$ is a finite integer or $-\infty$, and $b$ is a finite integer or $+\infty$ with $b-a \geq 3 ; \Delta$ and $\nabla$ are the forward and backward difference operators, respectively, i.e., $\Delta x(t)=x(t+1)-x(t)$ and $\nabla x(t)=x(t)-x(t-1) ; p(t)$ and $q(t)$ are complex with $p(t) \neq 0$ for $t \in I, p(a-1) \neq 0$ if $a$ is finite and $p(b+1) \neq 0$ if $b$ is finite; $w(t)>0$ for $t \in I$; and $\lambda$ is a spectral parameter.

Equation (1.1) is formally symmetric if and only if both $p(t)$ and $q(t)$ are real numbers. Therefore, if $p(t)$ or $q(t)$ are complex, then Eq. (1.1) is formally nonsymmetric. To study nonsymmetric operators, Glazman introduced the concept of $J$-symmetric operators in [1] where $J$ is a conjugation operator (see Definition 2.2). The minimal operators generated by Sturm-Liouville and some higher-order differential and difference expressions with complex coefficients are $J$-symmetric operators in the related Hilbert spaces (e.g., $[2-4])$. Here, we remark that a bounded $J$-symmetric operator is also called a complex symmetric operator $(c f .[5,6])$. The operators generated by singular differential and difference expressions are not bounded in general.

It is well known that the study of spectra of symmetric $(J$-symmetric) differential expressions is to consider the spectra of self-adjoint ( $J$-self-adjoint) operators generated by such expressions. In general, under a certain definiteness condition, a formally differential expression can generate a minimal operator in a related Hilbert space and its adjoint 
is the corresponding maximal operator (see, e.g., $[7,8])$. Generally, the self-adjoint $(J$ self-adjoint) operators are generated by extending the minimal operators. In addition, the eigenvalues of every self-adjoint ( $J$-self-adjoint) extension of the corresponding minimal operator are different although the essential spectra of them are the same. Therefore, the characterization of self-adjoint ( $J$-self-adjoint) extensions of a differential expression is a primary task in the study of its spectral problems; and the classical von Neumann selfadjoint extension theory and the Glazman-Krein-Naimark (GKN) theory for symmetric operators were established $[9,10]$. The related $J$-self-adjoint extension theory was also established $(c f .[3,11])$. By using them, characterizations of self-adjoint ( $J$-self-adjoint) extensions for differential expressions in terms of boundary conditions have been given ( $c f$. $[4,7,12,13])$. For other results for formally symmetric $(J$-symmetric) differential expressions, the reader is referred to [14-22] and the references therein.

It has been found out that the minimal operators generated by some differential expressions may be non-densely defined and the maximal operators may be multi-valued (e.g., see [20, Example 2.2]). In particular, the maximal operator corresponding to Eq. (1.1) is multi-valued, and the minimal operator is non-densely defined in the related Hilbert space (cf. [23]). Therefore, the self-adjoint extension theory for symmetric operators is not applicable in these cases. Coddington [24] extended the von Neumann self-adjoint extension theory for symmetric operators to Hermitian subspaces in 1973. Recently, Shi [25] extended the GKN theory for symmetric operators to Hermitian subspaces. Using GKN theory given in [25], Shi [23] first studied the self-adjoint extensions of (1.1) with real coefficients in the framework of subspaces in a product space. For $J$-symmetric case, in order to study the $J$-self-adjoint extensions of $J$-symmetric differential and difference expressions for which the minimal operators are non-densely defined or the maximal operators are multi-valued, the theory for a $J$-Hermitian subspace was given in [26] which includes the GKN theorem for a $J$-Hermitian subspace. For the results for difference expressions, the reader is referred to [27-33].

The limit types of (1.1) which are directly related to how many boundary conditions should be added to get a $J$-self-adjoint extension have been investigated in [31, 32]. In the present paper, the $J$-self-adjoint subspace extensions and $J$-self-adjoint operator extensions of the minimal subspace corresponding to Eq. (1.1) with complex coefficients are studied. A complete characterization of them in terms of boundary conditions is given. These characterizations are basic in the study of spectral theory for Eq. (1.1).

The rest of this present paper is organized as follows. In Section 2, some basic concepts and fundamental results about subspaces and Eq. (1.1) are introduced. In Section 3, the maximal, pre-minimal, and minimal subspaces in the whole interval and the left-hand and right-hand half-intervals are introduced and their properties are studied. The relationship among the defect indices of the minimal subspaces in the whole interval and the left-hand and right-hand half-intervals is studied in Section 4. In Section 5, we pay our attention to $J$-self-adjoint subspace extensions of the minimal subspace in the whole interval. Finally, a complete characterization of $J$-self-adjoint operator extensions of the minimal operator in the whole interval is given in Section 6. Three examples are given in Section 7.

\section{Preliminaries}

In this section, we introduce some basic concepts and give some fundamental results about subspaces in a product space and present two results about Eq. (1.1). 
By $\mathbf{C}$ denote the set of complex numbers, and by $\bar{z}$ denote the complex conjugate of $z \in \mathbf{C}$. Let $X$ be a complex Hilbert space with the inner product $\langle\cdot, \cdot\rangle$. The norm $\|\cdot\|$ is defined by $\|f\|=\langle f, f\rangle^{1 / 2}$ for $f \in X$. Let $X^{2}$ be the product space $X \times X$ with the following induced inner product, denoted by $\langle\cdot, \cdot\rangle$ without any confusion:

$$
\langle(x, f),(y, g)\rangle=\langle x, y\rangle+\langle f, g\rangle \quad \text { for all }(x, f),(y, g) \in X^{2} .
$$

Let $T$ be a linear subspace in $X^{2}$. For briefness, a linear subspace is only called a subspace. For a subspace $T$ in $X^{2}$, denote

$$
\begin{aligned}
& D(T)=\{x \in X:(x, f) \in T \text { for some } f \in X\}, \\
& T(x)=\{f \in X:(x, f) \in T\}, \\
& T-\lambda=\{(x, f-\lambda x):(x, f) \in T\} .
\end{aligned}
$$

Clearly, $T(0)=\{0\}$ if and only if $T$ can determine a unique linear operator from $D(T)$ into $X$ whose graph is $T$. Therefore, $T$ is said to be an operator if $T(0)=\{0\}$.

Definition 2.1 [24] Let $T$ be a subspace in $X^{2}$.

(1) Its adjoint, $T^{*}$, is defined by

$$
T^{*}=\left\{(y, g) \in X^{2}:\langle f, y\rangle=\langle x, g\rangle \text { for all }(x, f) \in T\right\} .
$$

(2) $T$ is said to be a Hermitian subspace if $T \subset T^{*}$.

(3) $T$ is said to be a self-adjoint subspace if $T=T^{*}$.

Lemma 2.1 [24] Let $T$ be a subspace in $X^{2}$. Then $T^{*}$ is a closed subspace in $X^{2}, T^{*}=(\bar{T})^{*}$, and $T^{* *}=\bar{T}$, where $\bar{T}$ is the closure of $T$.

Definition 2.2 (see [19, p.114] or [3]) An operator $J$ defined on $X$ is said to be a conjugation operator if for all $x, y \in X$,

$$
\langle J x, J y\rangle=\langle y, x\rangle \text { and } J^{2} x=x
$$

It can be verified that $J$ is a conjugate linear, norm-preserving bijection on $X$ and it holds that (see [19, p.114])

$$
\langle J x, y\rangle=\langle J y, x\rangle \quad \text { for all } x, y \in X .
$$

The complex conjugation $x \mapsto \bar{x}$ in any $l^{2}$ space is a conjugation operator on $l^{2}$.

Definition 2.3 [26] Let $T$ be a subspace in $X^{2}$ and $J$ be a conjugation operator.

(1) The $J$-adjoint of $T$, i.e., $T_{J}^{*}$, is defined by

$$
T_{J}^{*}=\left\{(y, g) \in X^{2}:\langle f, J y\rangle=\langle x, J g\rangle \text { for all }(x, f) \in T\right\} .
$$

(2) $T$ is said to be a $J$-Hermitian subspace if $T \subset T_{J}^{*}$. 
(3) $T$ is said to be a $J$-self-adjoint subspace if $T=T_{J}^{*}$.

(4) Let $T$ be a $J$-Hermitian subspace. Then $S$ is a $J$-self-adjoint subspace extension (briefly, $J$-SSE) of $T$ if $T \subset S$ and $S$ is a $J$-self-adjoint subspace.

\section{Remark 2.1}

(i) It can be easily verified that $T_{J}^{*}$ is a closed subspace. Consequently, a $J$-self-adjoint subspace $T$ is a closed subspace since $T=T_{J}^{*}$. In addition, $S_{J}^{*} \subset T_{J}^{*}$ if $T \subset S$.

(ii) From the definition, we have that $\langle f, J y\rangle=\langle x, J g\rangle$ holds for all $(x, f) \in T$ and $(y, g) \in T_{J}^{*}$, and that $T$ is a $J$-Hermitian subspace if and only if

$$
\langle f, J y\rangle=\langle x, J g\rangle \quad \text { for all }(x, f),(y, g) \in T .
$$

(iii) Assume that $T$ is not only $J$-symmetric for some conjugation operator $J$ but also symmetric, and that $S$ is a $J$-SSE of $T$. Then $S$ is a self-adjoint subspace extension of $T$ if and only if $S_{J}^{*}=S^{*}$.

Lemma 2.2 [26] Let $T$ be a subspace in $X^{2}$. Then

(1) $T^{*}=\left\{(J y, J g):(y, g) \in T_{J}^{*}\right\}$;

(2) $T_{J}^{*}=\left\{(J y, J g):(y, g) \in T^{*}\right\}$.

Lemma 2.3 [26] Let $T$ be a J-Hermitian subspace. Then $(y, g) \in \bar{T}$ if and only if $(y, g) \in T_{J}^{*}$ and $\langle f, J y\rangle=\langle x, J g\rangle$ for all $(x, f) \in T_{J}^{*}$.

Definition 2.4 [26] Let $T$ be a $J$-Hermitian subspace. Then $d(T)=\frac{1}{2} \operatorname{dim} T_{J}^{*} / \bar{T}$ is called to be the defect index of $T$.

Remark 2.2 By [26, Remark 3.5], $d(T)$ is a nonnegative integer or else infinite. Further, $d(T)=d(\bar{T})$. Then $T$ and $\bar{T}$ have the same $J$-SSEs since every $J$-SSE is closed.

Define the form [:] as

$$
[(x, f):(y, g)]=\langle f, J y\rangle-\langle x, J g\rangle, \quad(x, f),(y, g) \in T_{J}^{*} .
$$

Then, for all $Y_{j}=\left(x_{j}, f_{j}\right) \in T_{J}^{*}(j=1,2,3)$ and $\mu \in \mathbf{C}$, it holds that

$$
\begin{array}{ll}
{\left[Y_{3}: Y_{1}+Y_{2}\right]=\left[Y_{3}: Y_{1}\right]+\left[Y_{3}: Y_{2}\right],} & {\left[Y_{1}+Y_{2}: Y_{3}\right]=\left[Y_{1}: Y_{3}\right]+\left[Y_{2}: Y_{3}\right],} \\
{\left[\mu Y_{1}: Y_{2}\right]=\left[Y_{1}: \mu Y_{2}\right]=\mu\left[Y_{1}: Y_{2}\right],} & {\left[Y_{1}: Y_{2}\right]=-\left[Y_{2}: Y_{1}\right] .}
\end{array}
$$

The following result which can be regarded as the GKN theorem for a $J$-Hermitian subspace was established in [26].

Theorem 2.1 Let $T$ be a closed J-Hermitian subspace. Assume that $d(T)=: d<+\infty$. Then a subspace $S$ is a J-SSE of $T$ if and only if $T \subset S \subset T_{J}^{*}$ and there exists $\left\{\left(x_{j}, f_{j}\right)\right\}_{j=1}^{d} \subset T_{J}^{*}$ such that

(i) $\left(x_{1}, f_{1}\right),\left(x_{2}, f_{2}\right), \ldots,\left(x_{d}, f_{d}\right)$ are linearly independent $(\operatorname{modulo} T)$;

(ii) $\left[\left(x_{s}, f_{s}\right):\left(x_{j}, f_{j}\right)\right]=0$ for $s, j=1,2, \ldots, d$;

(iii) $S=\left\{(y, g) \in T_{J}^{\prime \prime}:\left[(y, g):\left(x_{j}, f_{j}\right)\right]=0, j=1,2, \ldots, d\right\}$. 
Finally, we present two results for $\tau$ or Eq. (1.1). For briefness, introduce the conventions: for any given integer $k, a+k=-\infty$ when $a=-\infty$, and $b+k=+\infty$ when $b=+\infty$. Further, denote

$$
(x, y)(t)=p(t)[(\Delta y(t)) x(t)-y(t) \Delta x(t)], \quad t \in\{t\}_{t=a-1}^{b} .
$$

In the case of $a=-\infty$, if $\lim _{t \rightarrow a}(x, y)(t)$ exists and is finite, then denote the limit by $(x, y)(-\infty)$; and in the other case of $b=+\infty$, if $\lim _{t \rightarrow b}(x, y)(t)$ exists and is finite, then denote the limit by $(x, y)(\infty)$.

We remark that the notation $(x, y)(t)$ is also used in [23] where it is given by $(x, y)(t)=$ $p(t)[(\Delta \bar{y}(t)) x(t)-\bar{y}(t) \Delta x(t)]$. So, the expression of $(x, y)(t)$ in the present paper is different from that in [23].

It can be easily verified that the following result holds.

Lemma 2.4 For any $x=\{x(t)\}_{t=a-1}^{b+1}, y=\{y(t)\}_{t=a-1}^{b+1} \subset \mathbf{C}$, and for any $m, n \in I$ with $m \leq n$,

$$
\sum_{t=m}^{n}[y(t) \tau(x)(t)-\tau(y)(t) x(t)]=\left.(x, y)(t)\right|_{t=m-1} ^{n}
$$

The following result is a direct consequence of Lemma 2.4.

Lemma 2.5 For each $\lambda \in \mathbf{C}$, let $y$ and $z$ be any solutions of (1.1). Then, for any given $a-1 \leq$ $t_{0} \leq b$

$$
(y, z)(t)=(y, z)\left(t_{0}\right), \quad t \in I \text {. }
$$

\section{Maximal and minimal subspaces}

In this section, we introduce the corresponding maximal, pre-minimal, and minimal subspaces to $\tau$ in the whole interval and the left-hand and right-hand half-intervals and study their properties.

First, introduce the following space:

$$
l_{w}^{2}(I):=\left\{x=\{x(t)\}_{t=a-1}^{b+1} \subset \mathbf{C}: \sum_{t=a}^{b} w(t)|x(t)|^{2}<+\infty\right\} .
$$

Then $l_{w}^{2}(I)$ is a Hilbert space with the inner product

$$
\langle x, y\rangle:=\sum_{t=a}^{b} \bar{y}(t) w(t) x(t)
$$

Clearly, $x=y$ in $l_{w}^{2}(I)$ if and only if $x(t)=y(t), t \in I$, i.e., $\|x-y\|=0$, where $\|x\|=\langle x, x\rangle^{1 / 2}$.

The formally adjoint operator of $\tau$ is

$$
\tau^{+}(x)(t):=-\nabla(\bar{p}(t) \Delta x(t))+\bar{q}(t) x(t), \quad t \in I .
$$


Now, introduce the maximal subspace $H(\tau)$ and the pre-minimal subspace $H_{00}(\tau)$ in $l_{w}^{2}(I) \times l_{w}^{2}(I)$ corresponding to $\tau$ as follows.

$$
\begin{aligned}
H(\tau)=\left\{(x, f) \in l_{w}^{2}(I) \times l_{w}^{2}(I): \tau(x)(t)=w(t) f(t), t \in I\right\}, \\
H_{00}(\tau)=\left\{(x, f) \in H(\tau): \text { there exist two integers } \tilde{t}_{0}, t_{0} \in I \text { with } \tilde{t}_{0}<t_{0}\right. \\
\left.\quad \text { such that } x(t)=0 \text { for } t \leq \tilde{t}_{0} \text { and } t \geq t_{0}\right\} .
\end{aligned}
$$

The subspace $H_{0}(\tau):=\bar{H}_{00}(\tau)$ is called the minimal subspace corresponding to $\tau$.

The endpoints $a$ and $b$ may be finite or infinite. In order to characterize the J-SSEs of $H_{0}(\tau)$ in a unified form, we introduce the left and right maximal and minimal subspaces. Fix any integer $a+1<c_{0}<b$. Denote

$$
I_{1}:=\{t\}_{t=a}^{t=c_{0}-1}, \quad I_{2}:=\{t\}_{t=c_{0}}^{b},
$$

and by $\langle\cdot, \cdot\rangle,\langle\cdot, \cdot\rangle_{a},\langle\cdot, \cdot\rangle_{b},\|\cdot\|,\|\cdot\|_{a}$, and $\|\cdot\|_{b}$ denote the inner products and the norms of $l_{w}^{2}(I), l_{w}^{2}\left(I_{1}\right)$, and $l_{w}^{2}\left(I_{2}\right)$, respectively. For briefness, we still denote the inner products and norms of their product spaces $l_{w}^{2}(I) \times l_{w}^{2}(I), l_{w}^{2}\left(I_{1}\right) \times l_{w}^{2}\left(I_{1}\right)$, and $l_{w}^{2}\left(I_{2}\right) \times l_{w}^{2}\left(I_{2}\right)$ by the same notations as those for $l_{w}^{2}(I), l_{w}^{2}\left(I_{1}\right)$, and $l_{w}^{2}\left(I_{2}\right)$, respectively.

Let $H_{a}(\tau)$ and $H_{a, 00}(\tau)$ be the left maximal and pre-minimal subspaces defined as in (3.1) with $I$ replaced by $I_{1}$, respectively, and let $H_{b}(\tau)$ and $H_{b, 00}(\tau)$ be the right maximal and preminimal subspaces defined as in (3.1) with $I$ replaced by $I_{2}$, respectively. The subspaces $H_{a, 0}(\tau):=\bar{H}_{a, 00}(\tau)$ and $H_{b, 0}(\tau):=\bar{H}_{b, 00}(\tau)$ are called the left and right minimal subspaces corresponding to $\tau$, respectively. By Lemma 2.1, one has

$$
H_{0}^{*}(\tau)=H_{00}^{*}(\tau), \quad H_{a, 0}^{*}(\tau)=H_{a, 00}^{*}(\tau), \quad H_{b, 0}^{*}(\tau)=H_{b, 00}^{*}(\tau) .
$$

In the rest of the present paper, let $J$ be the complex conjugate $x \mapsto \bar{x}$, i.e., $J x=\bar{x}$. Then $J$ is a conjugation operator on $l_{w}^{2}(I)\left(\right.$ or $l_{w}^{2}\left(I_{1}\right)$ or $\left.l_{w}^{2}\left(I_{2}\right)\right)$. By Lemma 2.2 and (3.2), one has that

$$
\begin{aligned}
& \left(H_{0}(\tau)\right)_{J}^{*}=\left(H_{00}(\tau)\right)_{J}^{*}, \quad\left(H_{a, 0}(\tau)\right)_{J}^{*}=\left(H_{a, 00}(\tau)\right)_{J}^{*}, \\
& \left(H_{b, 0}(\tau)\right)_{J}^{*}=\left(H_{b, 00}(\tau)\right)_{J}^{*} .
\end{aligned}
$$

The rest of this section is divided into three parts.

\subsection{Properties of minimal subspaces and their adjoint and $J$-adjoint subspaces}

In this subsection, we study the properties of minimal subspaces $H_{0}(\tau), H_{a, 0}(\tau), H_{b, 0}(\tau)$ and their adjoint and $J$-adjoint subspaces.

First, we have the following result.

Lemma 3.1 (see [23, Lemma 3.1]) For each $a+1 \leq t_{0} \leq b-1$ (or $a+1 \leq t_{0} \leq c_{0}-2$ or $c_{0}+1 \leq$ $\left.t_{0} \leq b-1\right)$ and for each $\xi \in \mathbf{C}$, there exists $x \in D\left(H_{00}(\tau)\right)\left(\operatorname{or} D\left(H_{a, 00}(\tau)\right)\right.$ or $\left.D\left(H_{b, 00}(\tau)\right)\right)$ such that $x\left(t_{0}\right)=\xi$ and $x(t)=0$ for all $t \neq t_{0}$. 
Theorem 3.1 $H\left(\tau^{+}\right) \subset H_{00}^{*}(\tau), H_{a}\left(\tau^{+}\right) \subset H_{a, 00}^{*}(\tau), H_{b}\left(\tau^{+}\right) \subset H_{b, 00}^{*}(\tau)$, and

$$
\begin{aligned}
& H_{00}^{*}(\tau)=\left\{(x, f) \in l_{w}^{2}(I) \times l_{w}^{2}(I): \tau^{+}(x)(t)=w(t) f(t), a+1 \leq t \leq b-1\right\}, \\
& H_{a, 00}^{*}(\tau)=\left\{(x, f) \in l_{w}^{2}\left(I_{1}\right) \times l_{w}^{2}\left(I_{1}\right): \tau^{+}(x)(t)=w(t) f(t), a+1 \leq t \leq c_{0}-2\right\}, \\
& H_{b, 00}^{*}(\tau)=\left\{(x, f) \in l_{w}^{2}\left(I_{2}\right) \times l_{w}^{2}\left(I_{2}\right): \tau^{+}(x)(t)=w(t) f(t), c_{0}+1 \leq t \leq b-1\right\} .
\end{aligned}
$$

Proof Since $H_{a, 00}(\tau)$ and $H_{b, 00}(\tau)$ are two special cases of $H_{00}(\tau)$, we only prove the results corresponding to $H_{00}(\tau)$.

For any given $(x, f) \in H_{00}^{*}(\tau)$, we have

$$
\langle f, y\rangle=\langle x, g\rangle, \quad \forall(y, g) \in H_{00}(\tau)
$$

which implies that

$$
\sum_{t=a}^{b}\left[\bar{y}(t) w(t) f(t)-\tau^{+}(\bar{y})(t) x(t)\right]=0 .
$$

On the other hand, by using $y \in D\left(H_{00}(\tau)\right)$, it can be verified that

$$
\sum_{t=a}^{b}\left[\bar{y}(t) \tau^{+}(x)(t)-\tau^{+}(\bar{y})(t) x(t)\right]=0
$$

which, together with (3.6) and $y(a)=y(b)=0$ when $a$ and $b$ are finite, implies that

$$
\sum_{t=a+1}^{b-1} \bar{y}(t)\left[w(t) f(t)-\tau^{+}(x)(t)\right]=0, \quad \forall y \in D\left(H_{00}(\tau)\right) .
$$

So, by Lemma 3.1 we get

$$
\tau^{+}(x)(t)=w(t) f(t), \quad a+1 \leq t \leq b-1 .
$$

Conversely, suppose that $(x, f) \in l_{w}^{2}(I) \times l_{w}^{2}(I)$ satisfies (3.7). Then (3.5) holds for all $(y, g) \in$ $H_{00}(\tau)$. Consequently, $(x, f) \in H_{00}^{*}(\tau)$. So, the first relation of (3.4) holds. In addition, the first relation of (3.4) directly yields that $H\left(\tau^{+}\right) \subset H_{00}^{*}(\tau)$. This completes the proof.

Theorem 3.2 The subspaces $H_{00}(\tau), H_{a, 00}(\tau)$, and $H_{b, 00}(\tau)$ are J-Hermitian subspaces in $l_{w}^{2}(I) \times l_{w}^{2}(I), l_{w}^{2}\left(I_{1}\right) \times l_{w}^{2}\left(I_{1}\right)$, and $l_{w}^{2}\left(I_{2}\right) \times l_{w}^{2}\left(I_{2}\right)$, respectively. Further, $H(\tau) \subset\left(H_{00}(\tau)\right)_{J}^{*}$, $H_{a}(\tau) \subset\left(H_{a, 00}(\tau)\right)_{J}^{*}$, and $H_{b}(\tau) \subset\left(H_{b, 00}(\tau)\right)_{J}^{*}$, and

$$
\begin{aligned}
& \left(H_{00}(\tau)\right)_{J}^{*}=\left\{(x, f) \in l_{w}^{2}(I) \times l_{w}^{2}(I): \tau(x)(t)=w(t) f(t), a+1 \leq t \leq b-1\right\}, \\
& \left(H_{a, 00}(\tau)\right)_{J}^{*}=\left\{(x, f) \in l_{w}^{2}\left(I_{1}\right) \times l_{w}^{2}\left(I_{1}\right): \tau(x)(t)=w(t) f(t), a+1 \leq t \leq c_{0}-2\right\}, \\
& \left(H_{b, 00}(\tau)\right)_{J}^{*}=\left\{(x, f) \in l_{w}^{2}\left(I_{2}\right) \times l_{w}^{2}\left(I_{2}\right): \tau(x)(t)=w(t) f(t), c_{0}+1 \leq t \leq b-1\right\} .
\end{aligned}
$$

Proof It can be easily verified that $H_{00}(\tau), H_{a, 00}(\tau)$, and $H_{b, 00}(\tau)$ are $J$-Hermitian subspaces in the corresponding Hilbert spaces by (ii) of Remark 2.1 and Lemma 2.4. Further, (3.8) can be concluded from Theorem 3.1 and Lemma 2.2. This completes the proof. 
Using Theorem 3.2 and with a similar argument to [23, Corollary 3.1], we can get the following results.

Corollary 3.1 $H(\tau)=\left(H_{00}(\tau)\right)_{J}^{*}=\left(H_{0}(\tau)\right)_{J}^{*}, H_{a}(\tau)=\left(H_{a, 00}(\tau)\right)_{J}^{*}=\left(H_{a, 0}(\tau)\right)_{J}^{*}$, and $H_{b}(\tau)=$ $\left(H_{b, 00}(\tau)\right)_{J}^{*}=\left(H_{b, 0}(\tau)\right)_{J}^{*}$ in the sense of the norms $\|\cdot\|,\|\cdot\|_{a}$, and $\|\cdot\|_{b}$, respectively. Consequently, $H(\tau), H_{a}(\tau)$, and $H_{b}(\tau)$ are closed subspaces in $l_{w}^{2}(I) \times l_{w}^{2}(I), l_{w}^{2}\left(I_{1}\right) \times l_{w}^{2}\left(I_{1}\right)$, and $l_{w}^{2}\left(I_{2}\right) \times l_{w}^{2}\left(I_{2}\right)$, respectively.

Remark 3.1 $H(\tau)=\left(H_{00}(\tau)\right)_{J}^{*}=\left(H_{0}(\tau)\right)_{J}^{*}$ follows from (3.3) and the first relation of (3.8) in the special case that $a=-\infty$ and $b=+\infty$.

Now, we introduce the boundary forms on $l_{w}^{2}(I) \times l_{w}^{2}(I), l_{w}^{2}\left(I_{1}\right) \times l_{w}^{2}\left(I_{1}\right)$, and $l_{w}^{2}\left(I_{2}\right) \times l_{w}^{2}\left(I_{2}\right)$ as follows.

$$
\begin{aligned}
& {[:]: l_{w}^{2}(I) \times l_{w}^{2}(I) \times l_{w}^{2}(I) \times l_{w}^{2}(I) \rightarrow \mathbf{C}, \quad((x, f),(y, g)) \mapsto\langle f, J y\rangle-\langle x, J g\rangle ;} \\
& {[:]_{a}: l_{w}^{2}\left(I_{1}\right) \times l_{w}^{2}\left(I_{1}\right) \times l_{w}^{2}\left(I_{1}\right) \times l_{w}^{2}\left(I_{1}\right) \rightarrow \mathbf{C}, \quad((x, f),(y, g)) \mapsto\langle f, J y\rangle_{a}-\langle x, J g\rangle_{a} ;} \\
& {[:]_{b}: l_{w}^{2}\left(I_{2}\right) \times l_{w}^{2}\left(I_{2}\right) \times l_{w}^{2}\left(I_{2}\right) \times l_{w}^{2}\left(\left(I_{2}\right) \rightarrow \mathbf{C}, \quad((x, f),(y, g)) \mapsto\langle f, J y\rangle_{b}-\langle x, J g\rangle_{b} .\right.}
\end{aligned}
$$

It can be easily shown that (2.3) holds for [:], [: $]_{a}$, and [:] $]_{b}$, respectively.

Note that $H_{0}(\tau), H_{a, 0}(\tau)$, and $H_{b, 0}(\tau)$ are closed. Then, by Lemma 2.3 and (3.3), $H_{0}(\tau)$, $H_{a, 0}(\tau)$, and $H_{b, 0}(\tau)$ can be expressed in terms of the boundary forms as follows.

$$
\begin{aligned}
& H_{0}(\tau)=\left\{(x, f) \in\left(H_{00}(\tau)\right)_{J}^{*}:\left[(x, f):\left(H_{00}(\tau)\right)_{J}^{*}\right]=0\right\}, \\
& H_{a, 0}(\tau)=\left\{(x, f) \in\left(H_{a, 00}(\tau)\right)_{J}^{*}:\left[(x, f):\left(H_{a, 00}(\tau)\right)_{J}^{*}\right]_{a}=0\right\}, \\
& H_{b, 0}(\tau)=\left\{(x, f) \in\left(H_{b, 00}(\tau)\right)_{J}^{*}:\left[(x, f):\left(H_{b, 00}(\tau)\right)_{J}^{*}\right]_{b}=0\right\} .
\end{aligned}
$$

Theorem 3.3 The subspaces $H_{0}(\tau), H_{a, 0}(\tau)$, and $H_{b, 0}(\tau)$ are closed J-Hermitian operators in $l_{w}^{2}(I), l_{w}^{2}\left(I_{1}\right)$, and $l_{w}^{2}\left(I_{2}\right)$, respectively.

Proof We only prove the result for $H_{0}(\tau)$ since $H_{a, 0}(\tau)$ and $H_{b, 0}(\tau)$ can be regarded as two special cases of $H_{0}(\tau)$.

Since $H_{0}(\tau)$ is a $J$-Hermitian subspace by Theorem 3.2 and $H_{0}(\tau)=\bar{H}_{00}(\tau)$, one has that $H_{0}(\tau)$ is a closed $J$-Hermitian subspace. So, it suffices to show that $\left(H_{0}(\tau)\right)(0)=\{0\}$. Suppose that $(0, f) \in H_{0}(\tau)$. Then, for all $(y, g) \in H(\tau) \subset\left(H_{00}(\tau)\right)_{J}^{*},[(0, f):(y, g)]=\langle f, J y\rangle=$ 0 , that is,

$$
\sum_{t=a}^{b} y(t) w(t) f(t)=0
$$

In order to show $f=0$, the discussion is divided into three cases.

Case 1. The endpoints $a$ and $b$ are finite. For all $(x, f) \in\left(H_{00}(\tau)\right)_{J}^{*}$ with $x(t)=0$ for all $t \in I$, we get by Theorem 3.2 and (3.10) that

$$
y(a) w(a) f(a)+y(b) w(b) f(b)=0, \quad \forall(y, g) \in H(\tau) .
$$


It can be easily shown that there exists $(y, g) \in H(\tau)$ such that $y(a)=f(a)$ and $y(t)=0$ for all $t \neq a$. Inserting it into (3.11) yields $f(a)=0$. Similarly, $f(b)=0$. Hence, $f=0$.

Case 2. One of $a$ and $b$ is finite. With a similar argument to that for Case 1 , one can show $f(a)=0$. Hence, $f=0$.

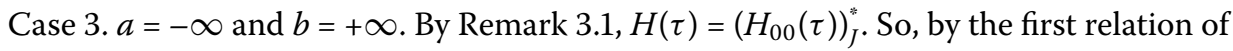
(3.8), $x(t)=0$ for $t \in I$ implies that $f(t)=0$ for $t \in I$. This completes the proof.

Lemma 3.2 For every $(x, f) \in H_{0}(\tau), x(a)=0$ in the case that $a$ is finite and $x(b)=0$ in the case that $b$ is finite.

Proof Fix any $(x, f) \in H_{0}(\tau)$. Then we have

$$
0=[(x, f):(y, g)]=\sum_{t=a}^{b} y(t) w(t) f(t)-\sum_{t=a}^{b} g(t) w(t) x(t), \quad \forall(y, g) \in H(\tau) .
$$

If $a$ is finite, then there exists $\left(y_{0}, g_{0}\right) \in H(\tau)$ such that $y_{0}(a-1) \neq 0$ and $y_{0}(t)=0$ for all $t \in I$. Inserting $\left(y_{0}, g_{0}\right)$ into (3.12), we have that $p(a-1) y_{0}(a-1) x(a)=0$. So, $x(a)=0$. One can get that $x(b)=0$ when $b$ is finite similarly. This completes the proof.

Theorem 3.4 The subspace $H_{0}(\tau)$ is a densely defined J-Hermitian operator in $l_{w}^{2}(I)$ in the case that $a=-\infty$ and $b=+\infty$ and a non-densely defined $J$-Hermitian operator in $l_{w}^{2}(I)$ in the case that at least one of $a$ and $b$ is finite. Consequently, $H_{a, 0}(\tau)$ and $H_{b, 0}(\tau)$ are nondensely defined J-Hermitian operators in $l_{w}^{2}\left(I_{1}\right)$ and $l_{w}^{2}\left(I_{2}\right)$, respectively.

Proof By Theorem 3.3, Lemma 3.2, and a similar method to [23, Theorem 3.3], this theorem can be proved.

\subsection{Characterizations of the three subspaces $\widehat{H}_{0}(\tau), \widehat{H}_{a, 0}(\tau)$, and $\widehat{H}_{b, 0}(\tau)$}

In this section, we introduce three subspaces $\widehat{H}_{0}(\tau), \widehat{H}_{a, 0}(\tau)$, and $\widehat{H}_{b, 0}(\tau)$, and discuss their characterizations, which will play an important role in the study of $J$-SSEs of $H_{0}(\tau)$.

First, define $\widehat{H}_{0}(\tau), \widehat{H}_{a, 0}(\tau)$, and $\widehat{H}_{b, 0}(\tau)$ in $l_{w}^{2}(I) \times l_{w}^{2}(I), l_{w}^{2}\left(I_{1}\right) \times l_{w}^{2}\left(I_{1}\right)$, and $l_{w}^{2}\left(I_{2}\right) \times l_{w}^{2}\left(I_{2}\right)$ as follows:

$$
\begin{aligned}
& \widehat{H}_{0}(\tau):=\{(x, f) \in H(\tau):[(x, f): H(\tau)]=0\}, \\
& \widehat{H}_{a, 0}(\tau):=\left\{(x, f) \in H_{a}(\tau):\left[(x, f): H_{a}(\tau)\right]_{a}=0\right\}, \\
& \widehat{H}_{b, 0}(\tau):=\left\{(x, f) \in H_{b}(\tau):\left[(x, f): H_{b}(\tau)\right]_{b}=0\right\} .
\end{aligned}
$$

Since [:], [:] $]_{a}$, and $[:]_{b}$ are defined in terms of the norms $\|\cdot\|,\|\cdot\|_{a}$, and $\|\cdot\|_{b}$, respectively, by Corollary 3.1 we get that $\widehat{H}_{0}(\tau)=H_{0}(\tau), \widehat{H}_{a, 0}(\tau)=H_{a, 0}(\tau)$, and $\widehat{H}_{b, 0}(\tau)=H_{b, 0}(\tau)$ in the sense of the norms $\|\cdot\|,\|\cdot\|_{a}$, and $\|\cdot\|_{b}$, respectively. So, $\widehat{H}_{0}(\tau), \widehat{H}_{a, 0}(\tau)$, and $\widehat{H}_{b, 0}(\tau)$ are closed $J$-Hermitian operators in the corresponding spaces by Theorem 3.3.

In [23], the patching lemma [23, Lemma 3.3] was used in the study of the self-adjoint subspace extensions for (1.1) with real coefficients. It also holds for (1.1) with complex coefficients here. 
Lemma 3.3 [23, Lemma 3.3] For any given $\alpha_{j}, \beta_{j} \in \mathbf{C}, j=1,2$, and any given $a_{1}, b_{1} \in I$ with $b_{1} \geq a_{1}+1$, there exists $f=\{f(t)\}_{t=a_{1}}^{b_{1}} \subset \mathbf{C}$ such that the boundary value problem

$$
\begin{aligned}
& \tau(x)(t)=w(t) f(t), \quad a_{1} \leq t \leq b_{1}, \\
& x\left(a_{1}-1\right)=\alpha_{1}, \quad x\left(a_{1}\right)=\alpha_{2}, \quad x\left(b_{1}\right)=\beta_{1}, \quad x\left(b_{1}+1\right)=\beta_{2}
\end{aligned}
$$

has a solution $x=\{x(t)\}_{t=a_{1}-1}^{b_{1}+1}$. Further, for any given $\left(x_{1}, f_{1}\right),\left(x_{2}, f_{2}\right) \in H(\tau)$, there exists $(y, g) \in H(\tau)$ such that

$$
y(t)=\left\{\begin{array}{ll}
x_{1}(t), & a-1 \leq t \leq a_{1}, \\
x_{2}(t), & b_{1} \leq t \leq b+1,
\end{array} \quad g(t)= \begin{cases}f_{1}(t), & a \leq t \leq a_{1}-1, \\
f_{2}(t), & b_{1}+1 \leq t \leq b .\end{cases}\right.
$$

Remark 3.2 (see [23, Remark 3.2]) Any two elements of $H_{a}(\tau)$ (or $H_{b}(\tau)$ ) can be patched together by some element of $H_{a}(\tau)$ (or $H_{b}(\tau)$ ) in a similar way as in Lemma 3.3. Further, any element of $H_{a}(\tau)$ and any element of $H_{b}(\tau)$ can be patched together by some element of $H(\tau)$ in a similar way as in Lemma 3.3.

The following result can be easily verified by Lemma 2.4 , Theorem 3.2, and (3.3).

Lemma 3.4 For all $x, y \in D\left(\left(H_{0}(\tau)\right)_{J}^{*}\right)$ or $D\left(\left(H_{a, 0}(\tau)\right)_{J}^{*}\right), \lim _{t \rightarrow a-1}(x, y)(t)$ exists and is finite in the case of $a=-\infty$, and for all $x, y \in D\left(\left(H_{0}(\tau)\right)_{J}^{*}\right)$ or $D\left(\left(H_{b, 0}(\tau)\right)_{J}^{*}\right), \lim _{t \rightarrow b}(x, y)(t)$ exists and is finite in the case of $b=+\infty$. Moreover,

$$
\begin{aligned}
& {[(x, f):(y, g)]=(x, y)(b)-(x, y)(a-1), \quad \forall(x, f),(y, g) \in H(\tau),} \\
& {[(x, f):(y, g)]_{a}=(x, y)\left(c_{0}-1\right)-(x, y)(a-1), \quad \forall(x, f),(y, g) \in H_{a}(\tau),} \\
& {[(x, f):(y, g)]_{b}=(x, y)(b)-(x, y)\left(c_{0}-1\right), \quad \forall(x, f),(y, g) \in H_{b}(\tau) .}
\end{aligned}
$$

Using Lemma 3.3 and with a similar argument to [23, Theorem 3.4], we have the other characterizations of three subspaces $\widehat{H}_{0}(\tau), \widehat{H}_{a, 0}(\tau)$, and $\widehat{H}_{b, 0}(\tau)$.

\section{Theorem 3.5}

$$
\begin{aligned}
\widehat{H}_{0}(\tau)= & \{(x, f) \in H(\tau):(x, y)(a-1)=(x, y)(b)=0, \forall y \in D(H(\tau))\} . \\
\widehat{H}_{a, 0}(\tau)= & \left\{(x, f) \in H_{a}(\tau): x\left(c_{0}-1\right)=x\left(c_{0}\right)=0\right. \\
& \text { and } \left.(x, y)(a-1)=0, \forall y \in D\left(H_{a}(\tau)\right)\right\} . \\
\widehat{H}_{b, 0}(\tau)= & \left\{(x, f) \in H_{b}(\tau): x\left(c_{0}-1\right)=x\left(c_{0}\right)=0\right. \\
& \text { and } \left.(x, y)(b)=0, \forall y \in D\left(H_{b}(\tau)\right)\right\} .
\end{aligned}
$$

\subsection{Characterizations of the left and right maximal subspaces}

In this section, we characterize $H_{a}(\tau)$ and $H_{b}(\tau)$.

First, let $d, d_{a}$, and $d_{b}$ be the defect indices of $H_{0}(\tau), H_{a, 0}(\tau)$, and $H_{b, 0}(\tau)$, respectively. Then we have 
Lemma $3.5 d=\frac{1}{2} \operatorname{dim} \mathcal{D}, d_{b}=\frac{1}{2} \operatorname{dim} \mathcal{D}_{b}$, and $d_{a}=\frac{1}{2} \operatorname{dim} \mathcal{D}_{a}$, where

$$
\begin{aligned}
& \mathcal{D}:=\left\{(y, g) \in H(\tau): \tau^{+}\left(\frac{1}{w} \tau(y)\right)(t)=-w(t) y(t), \forall a+1 \leq t \leq b-1\right\}, \\
& \mathcal{D}_{b}:=\left\{(y, g) \in H_{b}(\tau): \tau^{+}\left(\frac{1}{w} \tau(y)\right)(t)=-w(t) y(t), \forall c_{0}+1 \leq t \leq b-1\right\}, \\
& \mathcal{D}_{a}:=\left\{(y, g) \in H_{a}(\tau): \tau^{+}\left(\frac{1}{w} \tau(y)\right)(t)=-w(t) y(t), \forall a+1 \leq t \leq c_{0}-2\right\} .
\end{aligned}
$$

Proof Since the proofs are similar, we only prove $d=\frac{1}{2} \operatorname{dim} \mathcal{D}$.

First, it can be verified that

$$
\operatorname{dim}\left(H_{0}(\tau)\right)_{J}^{*} / H_{0}(\tau)=\operatorname{dim} H(\tau) / \widehat{H}_{0}(\tau)
$$

Next, we prove that

$$
H(\tau)=\widehat{H}_{0}(\tau) \oplus \mathcal{D} \quad \text { (orthogonal sum) }
$$

Let $(y, g) \in H(\tau) \ominus \widehat{H}_{0}(\tau)$, where $\ominus$ denotes the orthogonal complement of $\widehat{H}_{0}(\tau)$ in $H(\tau)$. Then

$$
0=\langle(y, g),(x, f)\rangle=\langle y, x\rangle+\langle g, f\rangle, \quad \forall(x, f) \in \widehat{H}_{0}(\tau)
$$

which yields that $(g,-y) \in \widehat{H}_{0}^{*}(\tau)$. It can be easily verified that $\widehat{H}_{0}^{*}(\tau)=H_{0}^{*}(\tau)$. So, $(g,-y) \in$ $H_{0}^{*}(\tau)$, and by Theorem 3.1, one has that

$$
\tau^{+}(g)(t)=-w(t) y(t), \quad \forall a+1 \leq t \leq b-1
$$

Since $(y, g) \in H(\tau)$ and $w \neq 0$, we get $g=\frac{1}{w} \tau(y)$ on $I$. Inserting it into (3.16), we have

$$
\tau^{+}\left(\frac{1}{w} \tau(y)\right)(t)=-w(t) y(t), \quad \forall a+1 \leq t \leq b-1 .
$$

So, $(y, g) \in \mathcal{D}$. Conversely, suppose that $(y, g) \in \mathcal{D}$. Then $(y, g) \in H(\tau)$ and (3.17) holds, and then (3.16) holds. Then $(g,-y) \in H_{0}^{*}(\tau)$ and hence $(g,-y) \in \widehat{H}_{0}^{*}(\tau)$. So, (3.15) holds and hence $(y, g) \in H(\tau) \ominus \widehat{H}_{0}(\tau)$. So, (3.14) holds, which together with (3.13) implies that $d=$ $\frac{1}{2} \operatorname{dim} \mathcal{D}$. This completes the proof.

Lemma $3.6 d_{b}=1$ or 2 and $d_{a}=1$ or 2 .

Proof By Lemma 3.5, $\operatorname{dim} \mathcal{D}_{b}$ is equal to the number of linearly independent solutions of

$$
\tau^{+}\left(\frac{1}{w} \tau(y)\right)(t)=-w(t) y(t), \quad c_{0}+1 \leq t \leq b-1
$$

for which both $y$ and $\frac{1}{w} \tau y$ are in $l_{w}^{2}\left(I_{2}\right)$. Then $d_{b}=\frac{1}{2} \operatorname{dim} \mathcal{D}_{b} \leq 2$ since (3.18) has at most four linearly independent solutions. In addition, there exists $\left(z_{j}, h_{j}\right) \in H_{b}(\tau), j=1,2$, such 
that

$$
\begin{aligned}
& z_{1}\left(c_{0}-1\right)=1, \quad z_{1}\left(c_{0}\right)=0, \quad z_{1}(t)=0, \quad \forall c_{0}+1 \leq t \leq b+1, \\
& z_{2}\left(c_{0}-1\right)=0, \quad z_{2}\left(c_{0}\right)=1, \quad z_{2}(t)=0, \quad \forall c_{0}+1 \leq t \leq b+1 .
\end{aligned}
$$

Note that $\left(z_{1}, h_{1}\right)$ and $\left(z_{2}, h_{2}\right)$ are linearly independent (modulo $\left.\widehat{H}_{b, 0}(\tau)\right)$ and

$$
d_{b}=\frac{1}{2} \operatorname{dim} H_{b}(\tau) / \widehat{H}_{b, 0}(\tau)
$$

Then $d_{b} \geq 1$ and hence $1 \leq d_{b} \leq 2$. Then $d_{b}=1$ or 2 since $d_{b}$ is an integer.

The assertion $d_{a}=1$ or 2 can be proved similarly. This completes the proof.

\section{Lemma 3.7}

(1) If all the solutions of (1.1) restricted on $I_{2}$ are in $l_{w}^{2}\left(I_{2}\right)$ for some $\lambda_{0} \in \mathbf{C}$, then the same is true for all $\lambda \in \mathbf{C}$.

(2) If all the solutions of the equation

$$
\tau^{+}\left(\frac{1}{w} \tau(y)\right)(t)=\lambda w(t) y(t), \quad c_{0}+1 \leq t<b-1,
$$

are in $l_{w}^{2}\left(I_{2}\right)$ for some $\lambda_{0} \in \mathbf{C}$, then the same is true for all $\lambda \in \mathbf{C}$.

Proof The first result is [31, Lemma 2.2]. Now, we prove the assertion (2). Clearly, this result holds if $b$ is finite. So, we prove the case where $b=+\infty$. By setting

$$
\begin{aligned}
& x_{1}(t)=y(t), \quad x_{2}(t)=-\frac{1}{w(t)} \tau(y)(t), \\
& x_{3}(t)=-\bar{p}(t) \Delta\left(\frac{1}{w(t)} \tau(y)(t)\right), \quad x_{4}(t)=p(t) \Delta y(t),
\end{aligned}
$$

Eq. (3.20) can be rewritten as the following discrete Hamiltonian system:

$$
\tilde{J} \Delta Y(t)=(P(t)-\lambda W(t)) R(Y)(t), \quad c_{0} \leq t<\infty,
$$

where

$$
\begin{aligned}
& Y(t)=\left(\begin{array}{l}
x_{1}(t) \\
x_{2}(t) \\
x_{3}(t) \\
x_{4}(t)
\end{array}\right), \quad R(Y)(t)=\left(\begin{array}{c}
x_{1}(t+1) \\
x_{2}(t+1) \\
x_{3}(t) \\
x_{4}(t)
\end{array}\right), \quad P(t)=\left(\begin{array}{cc}
-C(t) & 0 \\
0 & B(t)
\end{array}\right), \\
& C(t)=\left(\begin{array}{cc}
0 & \bar{q}(t+1) \\
q(t+1) & w(t+1)
\end{array}\right), \quad B(t)=\left(\begin{array}{cc}
0 & 1 / p(t) \\
1 / \bar{p}(t) & 0
\end{array}\right), \quad \tilde{J}=\left(\begin{array}{cc}
0 & -I_{2 \times 2} \\
I_{2 \times 2} & 0
\end{array}\right),
\end{aligned}
$$

$I_{2 \times 2}$ is the $2 \times 2$ unit matrix, and $W(t)=\operatorname{diag}(w(t+1), 0,0,0)$. It is evident that the assumptions $\left(A_{1}\right)$ and $\left(A_{2}\right)$ of [27, Section 1] hold for (3.22). Let

$$
l_{W}^{2}:=\left\{Y=\{Y(t)\}_{t=c_{0}}^{\infty} \subset \mathbf{C}^{4}: \sum_{t=c_{0}}^{\infty} R(Y)^{*}(t) W(t) R(Y)(t)<+\infty\right\}
$$


with the inner product $\langle Y, Z\rangle_{W}=\sum_{t=c_{0}}^{\infty} R(Z)^{\prime \prime}(t) W(t) R(Y)(t)$, where $Y^{\prime \prime}(t)$ denotes the complex conjugate transpose of $Y(t)$. We have from [27, Theorem 5.5] that if there exists $\lambda_{0} \in \mathbf{C}$ such that all the solutions of (3.22) are in $l_{W}^{2}$, then the same is true for all $\lambda \in \mathbf{C}$. Hence, the assertion (2) of this lemma follows. This completes the proof.

Theorem 3.6 Let $\left(z_{j}, h_{j}\right) \in H_{b}(\tau)(j=1,2)$ be defined by (3.19). Then the following results hold:

(1) In the case of $d_{b}=1$, for any given $(x, f) \in H_{b}(\tau)$, there exist uniquely $\left(y_{0}, f_{0}\right) \in \widehat{H}_{b, 0}(\tau)$ and $c_{1}, c_{2} \in \mathbf{C}$ such that

$$
x(t)=y_{0}(t)+c_{1} z_{1}(t)+c_{2} z_{2}(t), \quad c_{0}-1 \leq t \leq b+1 .
$$

(2) In the case of $d_{b}=2$, let $\phi_{1}$ and $\phi_{2}$ be two linearly independent solutions of (1.1) restricted on $I_{2}$. Then $\phi_{1}$ and $\phi_{2}$ are in $l_{w}^{2}\left(I_{2}\right)$, and for any given $(x, f) \in H_{b}(\tau)$, there exist uniquely $\left(y_{0}, f_{0}\right) \in \widehat{H}_{b, 0}(\tau)$ and $c_{j}, d_{j} \in \mathbf{C}(j=1,2)$ such that

$$
x(t)=y_{0}(t)+c_{1} z_{1}(t)+c_{2} z_{2}(t)+d_{1} \phi_{1}(t)+d_{2} \phi_{2}(t), \quad c_{0}-1 \leq t \leq b+1 .
$$

Proof Since $\operatorname{dim} H_{b}(\tau) / \widehat{H}_{b, 0}(\tau)=2$ in the case of $d_{b}=1$, one has that $\left(z_{1}, h_{1}\right)$ and $\left(z_{2}, h_{2}\right)$ defined by (3.19) form a basis of $H_{b}(\tau) / \widehat{H}_{b, 0}(\tau)$. So, the first result holds.

In the case of $d_{b}=2$, one has that $\operatorname{dim} H_{b}(\tau) / \widehat{H}_{b, 0}(\tau)=4$. By Lemmas 3.5 and 3.7, all the solutions of (3.20) with $\lambda=0$ are in $l_{w}^{2}\left(I_{2}\right)$ and hence all the solutions of $\tau(y)(t)=0$ restricted on $I_{2}$ are in $l_{w}^{2}\left(I_{2}\right)$. So, all the solutions of (1.1) restricted on $I_{2}$ are in $l_{w}^{2}\left(I_{2}\right)$ by Lemma 3.7. Let $\phi_{1}$ and $\phi_{2}$ be two linearly independent solutions of (1.1). Then $\left(\phi_{1}, \lambda \phi_{1}\right)$, $\left(\phi_{2}, \lambda \phi_{2}\right) \in H_{b}(\tau)$. Set

$$
\Phi:=\left(\left(\phi_{j}, \phi_{k}\right)\left(c_{0}-1\right)\right)_{2 \times 2} .
$$

Then it can be concluded that $\operatorname{rank} \Phi=2$. On the other hand, $\left(z_{1}, h_{1}\right),\left(z_{2}, h_{2}\right),\left(\phi_{1}, \lambda \phi_{1}\right)$, and $\left(\phi_{2}, \lambda \phi_{2}\right)$ are linearly independent (modulo $\left.\widehat{H}_{b, 0}(\tau)\right)$. In fact, if

$$
\left(\sum_{j=1}^{2} c_{j} z_{j}+\sum_{j=1}^{2} c_{j+1} \phi_{j}, \sum_{j=1}^{2} c_{j} h_{j}+\lambda \sum_{j=1}^{2} c_{j+1} \phi_{j}\right) \in \widehat{H}_{b, 0}(\tau)
$$

then by Theorem 3.5 and $\phi_{1}, \phi_{2} \in D\left(H_{b}(\tau)\right)$,

$$
\left\{\begin{array}{l}
\sum_{j=1}^{2} c_{j} z_{j}\left(c_{0}-1\right)+\sum_{j=1}^{2} c_{j+1} \phi_{j}\left(c_{0}-1\right)=0, \\
\sum_{j=1}^{2} c_{j} z_{j}\left(c_{0}\right)+\sum_{j=1}^{2} c_{j+1} \phi_{j}\left(c_{0}\right)=0, \\
c_{3}\left(\phi_{1}, \phi_{1}\right)(b)+c_{4}\left(\phi_{2}, \phi_{1}\right)(b)=0, \\
c_{3}\left(\phi_{1}, \phi_{2}\right)(b)+c_{4}\left(\phi_{2}, \phi_{2}\right)(b)=0 .
\end{array}\right.
$$

This, together with Lemma 2.5 and $\operatorname{rank} \Phi=2$, implies that $c_{j}=0(1 \leq j \leq 4)$. Then $\left(z_{1}, h_{1}\right)$, $\left(z_{2}, h_{2}\right),\left(\phi_{1}, \lambda \phi_{1}\right)$, and $\left(\phi_{2}, \lambda \phi_{2}\right)$ form a basis of $H_{b}(\tau) / \widehat{H}_{b, 0}(\tau)$. So, (3.24) holds. This completes the proof.

Using a similar argument to Theorem 3.6, we can get the following result. 
Theorem 3.7 Let $\left(\tilde{z}_{j}, \tilde{h}_{j}\right) \in H_{a}(\tau)(j=1,2)$ be defined by

$$
\begin{aligned}
& \tilde{z}_{1}\left(c_{0}-1\right)=1, \quad \tilde{z}_{1}\left(c_{0}\right)=0, \quad \tilde{z}_{1}(t)=0, \quad \forall a-1 \leq t \leq c_{0}-2, \\
& \tilde{z}_{2}\left(c_{0}-1\right)=0, \quad \tilde{z}_{2}\left(c_{0}\right)=1, \quad \tilde{z}_{2}(t)=0, \quad \forall a-1 \leq t \leq c_{0}-2 .
\end{aligned}
$$

Then the following results hold:

(1) In the case of $d_{a}=1$, for any given $(x, f) \in H_{a}(\tau)$, there exist uniquely $\left(\tilde{y}_{0}, \tilde{f}_{0}\right) \in \widehat{H}_{a, 0}(\tau)$ and $\tilde{c}_{1}, \tilde{c}_{2} \in \mathbf{C}$ such that

$$
x(t)=\tilde{y}_{0}(t)+\tilde{c}_{1} \tilde{z}_{1}(t)+\tilde{c}_{2} \tilde{z}_{2}(t), \quad a-1 \leq t \leq c_{0}
$$

(2) In the case of $d_{a}=2$, let $\tilde{\phi}_{1}$ and $\tilde{\phi}_{2}$ be two linearly independent solutions of equation (1.1) restricted on $I_{1}$. Then $\tilde{\phi}_{1}$ and $\tilde{\phi}_{2}$ are in $l_{w}^{2}\left(I_{1}\right)$, and for any given $(x, f) \in H_{a}(\tau)$, there exist uniquely $\left(\tilde{y}_{0}, \tilde{f}_{0}\right) \in \widehat{H}_{a, 0}(\tau)$ and $\tilde{c}_{j}, \tilde{d}_{j} \in \mathbf{C}(j=1,2)$ such that

$$
x(t)=\tilde{y}_{0}(t)+\tilde{c}_{1} \tilde{z}_{1}(t)+\tilde{c}_{2} \tilde{z}_{2}(t)+\tilde{d}_{1} \tilde{\phi}_{1}(t)+\tilde{d}_{2} \tilde{\phi}_{2}(t), \quad a-1 \leq t \leq c_{0} .
$$

\section{Defect indices of $H_{0}(\tau)$}

The following is the main result of this section.

Theorem 4.1 Let $d, d_{a}$, and $d_{b}$ be the defect indices of $H_{0}(\tau), H_{a, 0}(\tau)$, and $H_{b, 0}(\tau)$, respectively. Then $d=d_{a}+d_{b}-2$.

It is evident that Theorem 4.1 holds in the case that at least one of $a$ and $b$ is finite. So, it is only needed to consider the case that $a=-\infty$ and $b=+\infty$. Before proving Theorem 4.1, we prove three lemmas in this case.

Lemma 4.1 $d=\frac{1}{2} \operatorname{dim} \widetilde{\mathcal{D}}, d_{b}=\frac{1}{2} \operatorname{dim} \widetilde{\mathcal{D}}_{b}$, and $d_{a}=\frac{1}{2} \operatorname{dim} \widetilde{\mathcal{D}}_{a}$, where

$$
\begin{aligned}
& \widetilde{\mathcal{D}}:=\left\{(y, g) \in\left(H_{0}(\tau)\right)_{J}^{*}:(g,-y) \in H_{0}^{*}(\tau)\right\}, \\
& \widetilde{\mathcal{D}}_{b}:=\left\{(y, g) \in\left(H_{b, 0}(\tau)\right)_{J}^{*}:(g,-y) \in H_{b, 0}^{*}(\tau)\right\}, \\
& \widetilde{\mathcal{D}}_{a}:=\left\{(y, g) \in\left(H_{a, 0}(\tau)\right)_{J}^{*}:(g,-y) \in H_{a, 0}^{*}(\tau)\right\} .
\end{aligned}
$$

Proof It can be easily verified that $\left(H_{0}(\tau)\right)_{J}^{*}=H_{0}(\tau) \oplus \widetilde{\mathcal{D}}$. This gives that $d=\frac{1}{2} \operatorname{dim} \widetilde{\mathcal{D}}$. The other two relations are proved similarly. This completes the proof.

For any given $(x, f) \in l_{w}^{2}(I) \times l_{w}^{2}(I)$, denote

$$
x^{-}:=\{x(t)\}_{t=-\infty}^{c_{0}}, \quad x^{+}:=\{x(t)\}_{t=c_{0}-1}^{\infty}, \quad f^{-}:=\{f(t)\}_{t=-\infty}^{c_{0}}, \quad f^{+}:=\{f(t)\}_{t=c_{0}-1}^{\infty} .
$$

Then we have the following result.

Lemma 4.2 Let $\widetilde{H}_{0}(\tau)$ be the restriction of $\widehat{H}_{0}(\tau)$ defined by

$$
\widetilde{H}_{0}(\tau)=\left\{(x, f) \in \widehat{H}_{0}(\tau): x\left(c_{0}-1\right)=x\left(c_{0}\right)=0\right\} .
$$


Then

$$
\begin{aligned}
\left(\widetilde{H}_{0}(\tau)\right)_{J}^{*}= & \left\{(y, g) \in l_{w}^{2}(I) \times l_{w}^{2}(I):\left(y^{-}, g^{-}\right) \in\left(H_{a, 0}(\tau)\right)_{J}^{*}\right. \text { and } \\
& \left.\left(y^{+}, g^{+}\right) \in\left(H_{b, 0}(\tau)\right)_{J}^{*}\right\} .
\end{aligned}
$$

Proof It can be easily verified by Theorem 3.5 that

$$
\begin{aligned}
& \widehat{H}_{a, 0}(\tau)=\left\{\left(x^{-}, f^{-}\right):(x, f) \in \widetilde{H}_{0}(\tau) \text { with } x^{+}(t) \equiv 0\right\}, \\
& \widehat{H}_{b, 0}(\tau)=\left\{\left(x^{+}, f^{+}\right):(x, f) \in \widetilde{H}_{0}(\tau) \text { with } x^{-}(t) \equiv 0\right\} .
\end{aligned}
$$

Then it can be verified that

$$
\begin{aligned}
\widetilde{H}_{0}^{*}(\tau)= & \left\{(y, g) \in l_{w}^{2}(I) \times l_{w}^{2}(I):\left(y^{-}, g^{-}\right) \in H_{a, 0}^{*}(\tau)\right. \text { and } \\
& \left.\left(y^{+}, g^{+}\right) \in H_{b, 0}^{*}(\tau)\right\} .
\end{aligned}
$$

Relation (4.1) follows from (4.3) and Lemma 2.2. This completes the proof.

Lemma 4.3 Let $\tilde{d}$ be the defect index of $\tilde{H}_{0}(\tau)$. Then $\tilde{d}=d_{a}+d_{b}$.

Proof It can be easily verified that $\widetilde{H}_{0}(\tau)$ is a closed $J$-Hermitian operator in $l_{w}^{2}(I)$ by the fact that $\widehat{H}_{0}(\tau)$ is a closed $J$-Hermitian operator in $l_{w}^{2}(I)$. Set

$$
\mathcal{D}_{a, b}=\left\{(y, g) \in l_{w}^{2}(I) \times l_{w}^{2}(I):\left(y^{-}, g^{-}\right) \in \widetilde{\mathcal{D}}_{a} \text { and }\left(y^{+}, g^{+}\right) \in \widetilde{\mathcal{D}}_{b}\right\}
$$

in which $\widetilde{\mathcal{D}}_{a}$ and $\widetilde{\mathcal{D}}_{b}$ are given in Lemma 4.1. Now, we prove that $\mathcal{D}_{a, b}=\left(\widetilde{H}_{0}(\tau)\right)_{J}^{\prime \prime} \ominus \widetilde{H}_{0}(\tau)$. Let $(y, g) \in\left(\widetilde{H}_{0}(\tau)\right)_{J}^{*} \ominus \widetilde{H}_{0}(\tau)$. Then, for all $(x, f) \in \widetilde{H}_{0}(\tau)$, (3.15) holds, which together with (4.2) implies that

$$
\left(g^{-},-y^{-}\right) \in \widehat{H}_{a, 0}^{*}(\tau), \quad\left(g^{+},-y^{+}\right) \in \widehat{H}_{b, 0}^{*}(\tau) .
$$

Since $H_{a, 0}^{*}(\tau)=\widehat{H}_{a, 0}^{*}(\tau)$ and $H_{b, 0}^{*}(\tau)=\widehat{H}_{b, 0}^{*}(\tau)$, one has $(y, g) \in \mathcal{D}_{a, b}$. Conversely, suppose that $(y, g) \in \mathcal{D}_{a, b}$. It can be verified that $(y, g) \in\left(\widetilde{H}_{0}(\tau)\right)_{J}^{*} \ominus \widetilde{H}_{0}(\tau)$ by (4.2). Hence, $\mathcal{D}_{a, b}=\left(\widetilde{H}_{0}(\tau)\right)_{J}^{*} \ominus \widetilde{H}_{0}(\tau)$. Therefore, $\tilde{d}=\frac{1}{2} \operatorname{dim} \mathcal{D}_{a, b}$. It can be easily verified that $\operatorname{dim} \mathcal{D}_{a, b}=$ $\operatorname{dim} \mathcal{D}_{a}+\operatorname{dim} \mathcal{D}_{b}$. So, $\tilde{d}=d_{a}+d_{b}$ by Lemma 4.1. This completes the proof.

Proof of Theorem 4.1 Set

$$
\widetilde{H}(\tau)=\left\{(x, f) \in H(\tau): x\left(c_{0}-1\right)=x\left(c_{0}\right)=0\right\} .
$$

There exist $\left(y_{1}, g_{1}\right),\left(y_{2}, g_{2}\right) \in H(\tau)$ such that

$$
\begin{aligned}
& y_{1}\left(c_{0}-1\right)=1, \quad y_{1}(t)=0, \quad \forall t \neq c_{0}-1, \\
& y_{2}\left(c_{0}\right)=1, \quad y_{2}(t)=0, \quad \forall t \neq c_{0} .
\end{aligned}
$$


Then $\left(y_{j}, g_{j}\right) \in \widehat{H}_{0}(\tau)$ by Theorem $3.5,\left(y_{j}, g_{j}\right) \notin \widetilde{H}_{0}(\tau)$, and $\left(y_{j}, g_{j}\right) \notin \widetilde{H}(\tau), j=1,2$. We claim that

$$
\begin{aligned}
& \widehat{H}_{0}(\tau)=\widetilde{H}_{0}(\tau) \dot{\operatorname{span}}\left\{\left(y_{1}, g_{1}\right),\left(y_{2}, g_{2}\right)\right\}, \\
& H(\tau)=\widetilde{H}(\tau)+\operatorname{span}\left\{\left(y_{1}, g_{1}\right),\left(y_{2}, g_{2}\right)\right\} .
\end{aligned}
$$

In fact, for each given $(x, f) \in \widehat{H}_{0}(\tau)$, the algebraic system

$$
\begin{aligned}
& c_{1} y_{1}\left(c_{0}-1\right)+c_{2} y_{2}\left(c_{0}-1\right)=x\left(c_{0}-1\right), \\
& c_{1} y_{1}\left(c_{0}\right)+c_{2} y_{2}\left(c_{0}\right)=x\left(c_{0}\right)
\end{aligned}
$$

has a unique solution $\left(\tilde{c}_{1}, \tilde{c}_{2}\right)^{T}$. Let $\tilde{x}=x-\left(\tilde{c}_{1} y_{1}+\tilde{c}_{2} y_{2}\right)$ and $\tilde{f}=f-\left(\tilde{c}_{1} g_{1}+\tilde{c}_{2} g_{2}\right)$. Then $(\tilde{x}, \tilde{f}) \in \widetilde{H}_{0}(\tau)$. So, every $(x, f) \in \widehat{H}_{0}(\tau)$ can be uniquely expressed as a linear combination of some element of $\widetilde{H}_{0}(\tau),\left(y_{1}, g_{1}\right)$, and $\left(y_{2}, g_{2}\right)$. Therefore, (4.4) holds. Similarly, (4.5) can be proved.

Furthermore, there exists $\left(x_{j}, f_{j}\right) \in\left(\widetilde{H}_{0}(\tau)\right)_{J}^{*}$ such that

$$
\begin{aligned}
& x_{j}(t)=\left\{\begin{array}{ll}
1, & t=c_{0}-1, \\
0, & t \neq c_{0}-1,
\end{array} \quad j=1,2, \quad x_{k}(t)=\left\{\begin{array}{ll}
1, & t=c_{0}, \\
0, & t \neq c_{0},
\end{array} \quad k=3,4,\right.\right.
\end{aligned} \quad \begin{aligned}
& f_{1}(t)=\left\{\begin{array} { l l } 
{ - \frac { p ( c _ { 0 } - 2 ) } { w ( c _ { 0 } - 2 ) } , } & { t = c _ { 0 } - 2 , } \\
{ \frac { r ( c _ { 0 } - 1 ) } { w ( c _ { 0 } - 1 ) } , } & { t = c _ { 0 } - 1 , } \\
{ - \frac { p ( c _ { 0 } - 1 ) } { w ( c _ { 0 } ) } , } & { t = c _ { 0 } , } \\
{ 0 , } & { t \neq c _ { 0 } - 2 , c _ { 0 } - 1 , c _ { 0 } , }
\end{array} \quad \left\{\begin{array}{ll}
-\frac{p\left(c_{0}-2\right)}{w\left(c_{0}-2\right)}, & t=c_{0}-2, \\
\frac{r\left(c_{0}-1\right)}{w\left(c_{0}-1\right)}, & t=c_{0}-1, \\
0, & t \neq c_{0}-2, c_{0}-1,
\end{array}\right.\right. \\
& f_{3}(t)=\left\{\begin{array}{ll}
-\frac{p\left(c_{0}\right)}{w\left(c_{0}+1\right)}, & t=c_{0}+1, \\
0, & t \neq c_{0}+1,
\end{array} \quad f_{4}(t)= \begin{cases}-\frac{p\left(c_{0}-1\right)}{w\left(c_{0}-1\right)}, & t=c_{0}-1, \\
-\frac{p\left(c_{0}\right)}{w\left(c_{0}+1\right)}, & t=c_{0}+1, \\
0, & t \neq c_{0}-1, c_{0}+1,\end{cases} \right.
\end{aligned}
$$

where $r(t):=p(t)+p(t-1)+q(t)$. Suppose that there exists $c_{j} \in \mathbf{C}$ such that $\sum_{j=1}^{4} c_{j}\left(x_{j}, f_{j}\right) \in$ $\widetilde{H}(\tau)$. Then we get from $w(t) \neq 0$ for $t \in I$ that

$$
\sum_{j=1}^{4} c_{j} x_{j}\left(c_{0}-1\right)=\sum_{j=1}^{4} c_{j} x_{j}\left(c_{0}\right)=0
$$

which implies that $\sum_{j=1}^{4} c_{j} x_{j}(t)=0, a-1 \leq t \leq b+1$. Therefore,

$$
\sum_{j=1}^{4} c_{j} f_{j}\left(c_{0}-1\right)=\sum_{j=1}^{4} c_{j} f_{j}\left(c_{0}\right)=0 .
$$

It can be obtained from (4.6) and (4.7) that $c_{j}=0$. So, $\left(x_{1}, f_{1}\right), \ldots,\left(x_{4}, f_{4}\right)$ are linearly independent (modulo $\widetilde{H}(\tau)$ ). Further, we claim that

$$
\left(\widetilde{H}_{0}(\tau)\right)_{J}^{*}=\widetilde{H}(\tau)+U
$$


where $U=\operatorname{span}\left\{\left(x_{1}, f_{1}\right),\left(x_{2}, f_{2}\right),\left(x_{3}, f_{3}\right),\left(x_{4}, f_{4}\right)\right\}$. In fact, it is evident that $\tilde{H}(\tau)+U \subset$ $\left(\widetilde{H}_{0}(\tau)\right)_{J}^{*}$. Now, we show $\left(\widetilde{H}_{0}(\tau)\right)_{J}^{*} \subset \widetilde{H}(\tau)+U$. For each given $(x, f) \in\left(\widetilde{H}_{0}(\tau)\right)_{J}^{*}$, the algebraic system

$$
\left\{\begin{array}{l}
\sum_{j=1}^{4} c_{j} x_{j}\left(c_{0}-1\right)=x\left(c_{0}-1\right) \\
\sum_{j=1}^{4} c_{j} x_{j}\left(c_{0}\right)=x\left(c_{0}\right) \\
\tau\left(\sum_{j=1}^{4} c_{j} x_{j}\right)\left(c_{0}-1\right)-w\left(c_{0}-1\right) \sum_{j=1}^{4} c_{j} f_{j}\left(c_{0}-1\right)=\tau(x)\left(c_{0}-1\right)-w\left(c_{0}-1\right) f\left(c_{0}-1\right) \\
\tau\left(\sum_{j=1}^{4} c_{j} x_{j}\right)\left(c_{0}\right)-w\left(c_{0}\right) \sum_{j=1}^{4} c_{j} f_{j}\left(c_{0}\right)=\tau(x)\left(c_{0}\right)-w\left(c_{0}\right) f\left(c_{0}\right)
\end{array}\right.
$$

has a unique solution $\left(\tilde{c}_{1}, \tilde{c}_{2}, \tilde{c}_{3}, \tilde{c}_{4}\right)^{T}$. Let $\tilde{x}=x-\sum_{j=1}^{4} \tilde{c}_{j} x_{j}$ and $\tilde{f}=f-\sum_{j=1}^{4} \tilde{c}_{j} f_{j}$. Then $(\tilde{x}, \tilde{f}) \in$ $\widetilde{H}(\tau)$. So, every $(x, f) \in\left(\widetilde{H}_{0}(\tau)\right)_{J}^{*}$ can be uniquely expressed as a linear combination of some element of $\widetilde{H}(\tau),\left(x_{1}, f_{1}\right), \ldots,\left(x_{4}, f_{4}\right)$. Therefore, $\left(\widetilde{H}_{0}(\tau)\right)_{J}^{*} \subset \widetilde{H}(\tau) \dot{+} U$ and hence (4.8) holds.

Since $\left(y_{1}, g_{1}\right)$ and $\left(y_{2}, g_{2}\right)$ are linearly independent (modulo $\widetilde{H}(\tau)$ ), it follows from (4.5) that $\operatorname{dim} H(\tau) / \widetilde{H}(\tau)=2$. Further, from (4.8),

$$
\operatorname{dim}\left(\widetilde{H}_{0}(\tau)\right)_{J}^{*} / \widetilde{H}(\tau)=4
$$

Then $\widetilde{H}(\tau) \subset H(\tau) \subset\left(\widetilde{H}_{0}(\tau)\right)_{J}^{*}$ implies

$$
\operatorname{dim}\left(\widetilde{H}_{0}(\tau)\right)_{J}^{*} / H(\tau)=2
$$

Since

$$
\widetilde{H}_{0}(\tau) \subset \widehat{H}_{0}(\tau) \subset H(\tau) \subset\left(\widetilde{H}_{0}(\tau)\right)_{J}^{*},
$$

we get from (3.13), (4.4), and (4.9) that

$$
\begin{aligned}
\tilde{d} & =\frac{1}{2} \operatorname{dim}\left(\widetilde{H}_{0}(\tau)\right)_{J}^{*} / \widetilde{H}_{0}(\tau) \\
& =\frac{1}{2}\left\{\operatorname{dim}\left(\widetilde{H}_{0}(\tau)\right)_{J}^{*} / H(\tau)+\operatorname{dim} H(\tau) / \widehat{H}_{0}(\tau)+\operatorname{dim} \widehat{H}_{0}(\tau) / \widetilde{H}_{0}(\tau)\right\} \\
& =2+d,
\end{aligned}
$$

which together with Lemma 4.3 implies that $d=d_{a}+d_{b}-2$. So, Theorem 4.1 holds. This completes the proof.

\section{$5 \mathrm{~J}$-self-adjoint subspace extensions of $\boldsymbol{H}_{\mathbf{0}}(\tau)$}

By [26, Theorem 4.3], $H_{0}(\tau)$ must have $J$-SSEs since it is $J$-Hermitian. In this section, we give a complete characterization of all the $J$-SSEs of $H_{0}(\tau)$ in terms of boundary conditions. This section consists of two subsections.

\subsection{The general case}

The discussion is divided into three cases: $d=0, d=1$, and $d=2$, which are equivalent to $d_{a}=d_{b}=1, d_{a}=1, d_{b}=2$ or $d_{a}=2, d_{b}=1$, and $d_{a}=d_{b}=2$, respectively, by Theorem 4.1.

The following result can be directly derived from Theorem 2.1 and Theorem 3.3.

Theorem 5.1 In the case of $d=0$, i.e., $d_{a}=d_{b}=1, H_{0}(\tau)$ is a J-self-adjoint operator. 
Theorem 5.2 In the case of $d=1$ with $d_{a}=2$ and $d_{b}=1$, let $\phi_{1}$ and $\phi_{2}$ be any two linearly independent solutions of (1.1). Then $H_{1}$ is a J-SSE of $H_{0}(\tau)$ (i.e., $H_{00}(\tau)$ ) if and only if there exists a matrix $M \in \mathbf{C}^{1 \times 2}$ such that $M \neq 0$ and

$$
H_{1}=\left\{(x, f) \in H(\tau): M\left(\begin{array}{l}
\left(x, \phi_{1}\right)(a-1) \\
\left(x, \phi_{2}\right)(a-1)
\end{array}\right)=0\right\} .
$$

Proof Note that $\phi_{1}^{-}, \phi_{2}^{-} \in l_{w}^{2}\left(I_{1}\right)$ by Theorem 3.7 and $b=+\infty$ in this case.

First, consider the sufficiency. Suppose that $M=\left(m_{1}, m_{2}\right) \neq 0$. Let $u=m_{1} \phi_{1}+m_{2} \phi_{2}$. It is evident that $u^{-} \in D\left(H_{a}(\tau)\right)$. Fix any integers $a_{1}$ and $b_{1}$ with $a<a_{1}+1<c_{0}<b_{1}-1$. By Remark 3.2, there exists $\beta=(y, g) \in H(\tau)$ such that

$$
y(t)= \begin{cases}u(t), & a-1 \leq t \leq a_{1}, \\ 0, & t \geq b_{1} .\end{cases}
$$

We claim that $\beta \notin H_{0}(\tau)$. Suppose on the contrary that $\beta \in H_{0}(\tau)$. Then $\beta \in \widehat{H}_{0}(\tau)$. Again by Remark 3.2, there exists $\left(y_{j}, g_{j}\right) \in H(\tau), j=1,2$, such that

$$
y_{j}(t)= \begin{cases}\phi_{j}(t), & a-1 \leq t \leq a_{1}, \\ 0, & t \geq b_{1} .\end{cases}
$$

So, we get from Lemma 3.4 and $\beta \in \widehat{H}_{0}(\tau)$ that

$$
0=\left(\left[\beta:\left(y_{1}, g_{1}\right)\right],\left[\beta:\left(y_{2}, g_{2}\right)\right]\right)=-M\left(\left(\phi_{j}, \phi_{k}\right)(a-1)\right)_{2 \times 2},
$$

which implies that $M=0$ since $\operatorname{rank}\left(\left(\phi_{j}, \phi_{k}\right)(a-1)\right)_{2 \times 2}=2$ from Lemma 2.5 and the proof of Theorem 3.6. This contradicts $M \neq 0$. Hence, $\beta \notin H_{0}(\tau)$. Note that $[\beta: \beta]=0$ and $d=1$. Then, by Theorem 2.1 and Corollary 3.1, the set

$$
H_{2}=\{F \in H(\tau):[F: \beta]=0\}
$$

is a $J$-SSE of $H_{0}(\tau)$. On the other hand, for any $F=(x, f) \in H(\tau)$, by Lemma 3.4 one has

$$
[(x, f): \beta]=-(x, y)(a-1)=-M\left(\begin{array}{l}
\left(x, \phi_{1}\right)(a-1) \\
\left(x, \phi_{2}\right)(a-1)
\end{array}\right),
$$

which implies that $H_{1}=H_{2}$. The sufficiency is shown.

Next, consider the necessity. Suppose that $H_{2}$ is a J-SSE of $H_{0}(\tau)$. By Theorem 2.1, Corollary 3.1, and $d=1$, there exists some element $\beta=(y, g) \in H(\tau)$ such that $\beta \notin H_{0}(\tau)$, $[\beta: \beta]=0$, and (5.3) holds. By (1) in Theorem 3.6 and (2) in Theorem 3.7, there exist uniquely $y_{b, 0} \in D\left(\widehat{H}_{b, 0}(\tau)\right)$ and uniquely $y_{a, 0} \in D\left(\widehat{H}_{a, 0}(\tau)\right)$ such that

$$
\begin{aligned}
& y(t)=y_{b, 0}(t)+c_{1} z_{1}(t)+c_{2} z_{2}(t), \quad c_{0}-1 \leq t \leq+\infty, \\
& y(t)=y_{a, 0}(t)+\sum_{j=1}^{2} \tilde{c}_{j} \tilde{z}_{j}(t)+\sum_{j=1}^{2} \tilde{d}_{j} \phi_{j}(t), \quad a-1 \leq t \leq c_{0},
\end{aligned}
$$


where $\tilde{c}_{k}, c_{k}, \tilde{d}_{k} \in \mathbf{C}$ and $z_{k}, \tilde{z}_{k}, k=1,2$, are defined by (3.19) and (3.26). If $\tilde{d}_{1}=\tilde{d}_{2}=0$, then it can be obtained from (5.4), (3.19), (3.26), Corollary 3.1, Lemma 3.4, and Theorem 3.5 that for all $(x, f) \in\left(H_{0}(\tau)\right)_{J}^{*}$ there exists $(\hat{x}, \hat{f}) \in H(\tau)$ such that

$$
[(x, f): \beta]=[(\hat{x}, \hat{f}): \beta]=(\hat{x}, y)(+\infty)-(\hat{x}, y)(a-1)=0 .
$$

So, $\beta \in H_{0}(\tau)$, which contradicts $\beta \notin H_{0}(\tau)$. Therefore, $\left|\tilde{d}_{1}\right|+\left|\tilde{d}_{2}\right|>0$. Set

$$
M:=\left(\tilde{d}_{1}, \tilde{d}_{2}\right)
$$

Then $M \neq 0$. Furthermore, for any $(x, f) \in H(\tau)$, by Lemma 3.4 one has

$$
[(x, f): \beta]=(x, y)(+\infty)-(x, y)(a-1)
$$

It follows from (5.4), (3.19), (3.26), and Theorem 3.5 that

$$
(x, y)(+\infty)=0, \quad(x, y)(a-1)=M\left(\begin{array}{l}
\left(x, \phi_{1}\right)(a-1) \\
\left(x, \phi_{2}\right)(a-1)
\end{array}\right) .
$$

So, $H_{2}$ determined by (5.3) can be expressed as (5.1). The necessity is proved. The entire proof is complete.

With a similar argument to Theorem 5.2, one can show the following result.

Theorem 5.3 In the case of $d=1$ with $d_{a}=1$ and $d_{b}=2$, let $\phi_{1}$ and $\phi_{2}$ be any two linearly independent solutions of (1.1). Then $H_{1}$ is a J-SSE of $H_{0}(\tau)$ (i.e., $\left.H_{00}(\tau)\right)$ if and only if there exists a matrix $N \in \mathbf{C}^{1 \times 2}$ such that $N \neq 0$ and

$$
H_{1}=\left\{(x, f) \in H(\tau): N\left(\begin{array}{l}
\left(x, \phi_{1}\right)(b) \\
\left(x, \phi_{2}\right)(b)
\end{array}\right)=0\right\} .
$$

Theorem 5.4 In the case of $d=2$, let $\phi_{1}$ and $\phi_{2}$ be any two linearly independent solutions of (1.1). Then $H_{1}$ is a J-SSE of $H_{0}(\tau)$ (i.e., $\left.H_{00}(\tau)\right)$ if and only if there exist two matrices $M, N \in \mathbf{C}^{2 \times 2}$ such that

$$
\begin{aligned}
& \operatorname{rank}(M, N)=2, \quad M \Phi M^{T}=N \Phi N^{T}, \\
& H_{1}=\left\{(x, f) \in H(\tau): M\left(\begin{array}{l}
\left(x, \phi_{1}\right)(a-1) \\
\left(x, \phi_{2}\right)(a-1)
\end{array}\right)-N\left(\begin{array}{l}
\left(x, \phi_{1}\right)(b) \\
\left(x, \phi_{2}\right)(b)
\end{array}\right)=0\right\},
\end{aligned}
$$

where $\Phi$ is defined by (3.25).

Proof Because $d=2$ is equivalent to $d_{a}=d_{b}=2$, it follows that $\phi_{1}^{-}$and $\phi_{2}^{-}$are in $l_{w}^{2}\left(I_{1}\right)$, and $\phi_{1}^{+}$and $\phi_{2}^{+}$are in $l_{w}^{2}\left(I_{2}\right)$ and hence $\phi_{1}$ and $\phi_{2}$ are in $l_{w}^{2}(I)$.

Step 1. Consider the sufficiency. Let $M=\left(m_{j k}\right), N=\left(n_{j k}\right)$, and

$$
\tilde{u}_{j}=\sum_{k=1}^{2} m_{j k} \phi_{k}, \quad u_{j}=\sum_{k=1}^{2} n_{j k} \phi_{k}, \quad j=1,2 .
$$


It is evident that $\tilde{u}_{j}, u_{j} \in H(\tau), j=1,2$. Choose any integers $a_{1}$ and $b_{1}$ with $a<a_{1}+1<c_{0}<$ $b_{1}-1<b$. By Lemma 3.3 there exists $\beta_{j}=\left(y_{j}, g_{j}\right) \in H(\tau)(j=1,2)$ such that

$$
y_{i}(t)= \begin{cases}\tilde{u}_{j}(t), & a-1 \leq t \leq a_{1} \\ u_{j}(t), & b_{1} \leq t \leq b+1\end{cases}
$$

By Theorem 3.5, $\operatorname{rank} \Phi=2$, and $\operatorname{rank}(M, N)=2$, it can be verified that $\beta_{1}$ and $\beta_{2}$ are linearly independent (modulo $H_{0}(\tau)$ ). Furthermore, by Lemmas 2.5 and 3.4, (5.6), and (5.8), we have

$$
\left(\left[\beta_{j}: \beta_{k}\right]\right)_{1 \leq j, k \leq 2}=N \Phi N^{T}-M \Phi M^{T}=0 .
$$

Therefore, by Theorem 2.1 and Corollary 3.1, it can be concluded that

$$
H_{2}=\left\{F \in H(\tau):\left[F: \beta_{j}\right]=0, j=1,2\right\}
$$

is a $J$-SSE of $H_{0}(\tau)$. For any $F=(x, f) \in H(\tau)$,

$$
\left(\begin{array}{l}
\left(x, y_{1}\right)(a-1) \\
\left(x, y_{2}\right)(a-1)
\end{array}\right)=M\left(\begin{array}{l}
\left(x, \phi_{1}\right)(a-1) \\
\left(x, \phi_{2}\right)(a-1)
\end{array}\right), \quad\left(\begin{array}{l}
\left(x, y_{1}\right)(b) \\
\left(x, y_{2}\right)(b)
\end{array}\right)=N\left(\begin{array}{l}
\left(x, \phi_{1}\right)(b) \\
\left(x, \phi_{2}\right)(b)
\end{array}\right)
$$

Lemma 3.4 and (5.10) yield that $H_{1}=H_{2}$. The sufficiency is proved.

Step 2. Consider the necessity. Suppose that $H_{2}$ is a $J$-SSE of $H_{0}(\tau)$. By Theorem 2.1 and Corollary 3.1, there exist two linearly independent (modulo $H_{0}(\tau)$ ) elements $\beta_{1}$ and $\beta_{2}$ in $H(\tau)$ such that $\left[\beta_{j}: \beta_{k}\right]=0, j, k=1,2$, and (5.9) holds. Note that $\beta_{j}=\left(y_{j}, g_{j}\right) \in H(\tau)$ and hence $\left(y_{j}^{-}, g_{j}^{-}\right) \in H_{a}(\tau)$ and $\left(y_{j}^{+}, g_{j}^{+}\right) \in H_{b}(\tau)$. By Theorems 3.7 and 3.6, there exist uniquely $\tilde{y}_{j 0} \in D\left(\widehat{H}_{a, 0}(\tau)\right), y_{j 0} \in D\left(\widehat{H}_{b, 0}(\tau)\right), \tilde{c}_{j k}, \tilde{n}_{j k}, c_{j k}, n_{j k} \in \mathbf{C}(j=1,2)$ such that

$$
\begin{aligned}
& y_{j}(t)=\tilde{y}_{j 0}(t)+\sum_{k=1}^{2} \tilde{c}_{j k} \tilde{z}_{k}(t)+\sum_{k=1}^{2} \tilde{n}_{j k} \phi_{k}(t), \quad a-1 \leq t \leq c_{0}, \\
& y_{j}(t)=y_{j 0}(t)+\sum_{k=1}^{2} c_{j k} z_{k}(t)+\sum_{k=1}^{2} n_{j k} \phi_{k}(t), \quad c 0-1 \leq t \leq b+1,
\end{aligned}
$$

where $\tilde{z}_{k}$ and $z_{k}$ are defined by (3.19) and (3.26). Set

$$
M=\left(\tilde{n}_{j k}\right), \quad N=\left(n_{j k}\right) .
$$

We will show that $\operatorname{rank}(M, N)=2$. Otherwise, $\operatorname{rank}(M, N)<2$. Then there exist $c_{1}, c_{2} \in \mathbf{C}$ with $\left|c_{1}\right|+\left|c_{2}\right|>0$ such that $\left(c_{1}, c_{2}\right)(M, N)=0$, i.e.,

$$
\left(c_{1}, c_{2}\right) M=\left(c_{1}, c_{2}\right) N=0 .
$$

Set $\beta=\left(y^{\prime}, g^{\prime}\right)=c_{1} \beta_{1}+c_{2} \beta_{2}$. Then $\beta \in H(\tau)$ and from (5.13) and Theorem 3.5,

$$
\begin{aligned}
& \left(\left(y^{\prime}, \phi_{1}\right)(a-1),\left(y^{\prime}, \phi_{2}\right)(a-1)\right)=\left(c_{1}, c_{2}\right) M\left(\left(\phi_{j}, \phi_{k}\right)(a-1)\right)_{2 \times 2}=0, \\
& \left(\left(y^{\prime}, \phi_{1}\right)(b),\left(y^{\prime}, \phi_{2}\right)(b)\right)=\left(c_{1}, c_{2}\right) N\left(\left(\phi_{j}, \phi_{k}\right)(b)\right)_{2 \times 2}=0 .
\end{aligned}
$$


By Theorems 3.6 and 3.7, for $y \in D(H(\tau)), y^{+}$can be uniquely expressed as (3.24) and $y^{-}$can be uniquely expressed as (3.28). So, it follows from (5.14) and Theorem 3.5 that $\left(y^{\prime}, y\right)(a-1)=\left(y^{\prime}, y\right)(b)=0$ for all $y \in D(H(\tau))$. This, together with Corollary 3.1 and Lemma 3.4, implies that $\left[\beta:\left(H_{00}(\tau)\right)_{J}^{*}\right]=[\beta: H(\tau)]=0$. Hence, $\beta \in H_{0}(\tau)$. Consequently, $\beta_{1}$ and $\beta_{2}$ are linearly dependent (modulo $H_{0}(\tau)$ ). This is a contradiction. So, $\operatorname{rank}(M, N)=2$. Further, from $\left[\beta_{j}: \beta_{k}\right]=0$ and Lemmas 2.5 and 3.4, (5.11), and Theorem 3.5, we get that

$$
0=\left(\left[\beta_{j}: \beta_{k}\right]\right)_{1 \leq j, k \leq 2}=N \Phi N^{T}-M \Phi M^{T} .
$$

So, $M$ and $N$ satisfy the second relation of (5.6).

Finally, for any $(x, f) \in H(\tau)$, it follows from (5.11) and Theorem 3.5 that (5.10) holds with $M$ and $N$ defined by (5.12). So, by Lemma 3.4, $H_{2}$ determined by (5.9) can be expressed as (5.7). The necessity is proved. The entire proof is complete.

\subsection{The special cases}

In this subsection, we characterize the $J$-SSEs of $H_{0}(\tau)$ in the special cases that one of the two endpoints $a$ and $b$ is finite and that both $a$ and $b$ are finite.

First, consider the case that $a$ is finite and $b=+\infty$. By Lemma $3.5, d_{a}=2$ in this case. Let $\phi_{1}$ and $\phi_{2}$ be two linearly independent solutions of (1.1) satisfying

$$
\begin{array}{ll}
\phi_{1}(a-1)=0, & p(a-1) \Delta \phi_{1}(a-1)=-1, \\
\phi_{2}(a-1)=1, & p(a-1) \Delta \phi_{2}(a-1)=0 .
\end{array}
$$

Then $\left(\left(\phi_{j}, \phi_{k}\right)(a-1)\right)_{2 \times 2}=-\hat{J}$ and hence by Lemma $2.5, \Phi=-\hat{J}$, where $\Phi$ is defined by (3.25) and $\hat{J}=\left(\begin{array}{ll}0 & -1 \\ 1 & 0\end{array}\right)$. It can be obtained from (5.15) that

$$
\left(\begin{array}{l}
\left(x, \phi_{1}\right)(a-1) \\
\left(x, \phi_{2}\right)(a-1)
\end{array}\right)=-\left(\begin{array}{c}
x(a-1) \\
p(a-1) \Delta x(a-1)
\end{array}\right) .
$$

Then the following result can be directly derived from Theorem 5.2.

Theorem 5.5 In the case that $a$ is finite, $b=+\infty$, and $d=1, H_{1}$ is a J-SSE of $H_{0}(\tau)$ (i.e., $\left.H_{00}(\tau)\right)$ if and only if there exists a matrix $M=\left(m_{1}, m_{2}\right) \in \mathbf{C}^{1 \times 2}$ with $M \neq 0$ such that

$$
H_{1}=\left\{(x, f) \in H(\tau): m_{1} x(a-1)+m_{2} p(a-1) \Delta x(a-1)=0\right\} .
$$

Furthermore, the following result is a direct consequence of (5.16), $\Phi=-\hat{J}$, and Theorem 5.4.

Theorem 5.6 In the case that $a$ is finite, $b=+\infty$, and $d=2$, let $\phi_{1}$ and $\phi_{2}$ be the solutions of (1.1) satisfying (5.15). Then $H_{1}$ is a J-SSE of $H_{0}(\tau)$ (i.e., $\left.H_{00}(\tau)\right)$ if and only if there exist matrices $M, N \in \mathbf{C}^{2 \times 2}$ such that

$$
\begin{aligned}
& \operatorname{rank}(M, N)=2, \quad M \hat{J} M^{T}=N \hat{\jmath} N^{T}, \\
& H_{1}=\left\{(x, f) \in H(\tau): M\left(\begin{array}{c}
x(a-1) \\
p(a-1) \Delta x(a-1)
\end{array}\right)+N\left(\begin{array}{l}
\left(x, \phi_{1}\right)(+\infty) \\
\left(x, \phi_{2}\right)(+\infty)
\end{array}\right)=0\right\} .
\end{aligned}
$$


Theorem 5.7 In the case that $a$ and $b$ are finite, $H_{1}$ is a J-SSE of $H_{0}(\tau)$ (i.e., $\left.H_{00}(\tau)\right)$ if and only if there exist matrices $M_{1}, N_{1} \in \mathbf{C}^{2 \times 2}$ such that

$$
\begin{aligned}
& \operatorname{rank}\left(M_{1}, N_{1}\right)=2, \quad M_{1} \hat{J} M_{1}^{T}=N_{1} \hat{J} N_{1}^{T}, \\
& H_{1}=\left\{(x, f) \in H(\tau): M_{1}\left(\begin{array}{c}
x(a-1) \\
p(a-1) \Delta x(a-1)
\end{array}\right)-N_{1}\left(\begin{array}{c}
x(b) \\
p(b) \Delta x(b)
\end{array}\right)=0\right\} .
\end{aligned}
$$

Proof In this case, $d_{a}=d_{b}=2$. Let $\phi_{1}$ and $\phi_{2}$ be the solutions of (1.1) satisfying (5.15). Then $\Phi=-\hat{J}$. By Theorem 5.4, $H_{1}$ is a $J$-SSE of $H_{0}(\tau)$ if and only if there exist matrices $M, N \in \mathbf{C}^{2 \times 2}$ such that (5.6) and (5.7) hold. Set

$$
M_{1}=-M, \quad N_{1}=N P^{T} \hat{J}, \quad P=\left(\begin{array}{cc}
\phi_{1}(b) & \phi_{2}(b) \\
p(b) \Delta \phi_{1}(b) & p(b) \Delta \phi_{2}(b)
\end{array}\right) .
$$

Then $P$ is invertible and hence $\operatorname{rank}\left(M_{1}, N_{1}\right)=\operatorname{rank}(M, N)$. It can be verified that

$$
N_{1} \hat{J} N_{1}^{T}=N \hat{J} N^{T}, \quad N_{1}\left(\begin{array}{c}
\left(x, \phi_{1}\right)(b) \\
\left(x, \phi_{2}\right)(b)
\end{array}\right)=N\left(\begin{array}{c}
x(b) \\
p(b) \Delta x(b)
\end{array}\right) .
$$

So, (5.6) and (5.7) hold if and only if (5.17) and (5.18) hold by (5.16). This completes the proof.

Remark 5.1 Let $p$ and $q$ be real-valued. Then $H_{0}(\tau)$ is not only $J$-symmetric but also symmetric. However, the set of all the J-SSEs is not equal to the set of all the SSEs (SSE is an abbreviation of self-adjoint subspace extension) in general, except for the case that $d=0$. For example, let $a$ be finite, $b=+\infty$, and $d=1$, and set

$$
H_{1}=\{(x, f) \in H(\tau):(1+i) x(a-1)+p(a-1) \Delta x(a-1)=0\} .
$$

Then $H_{1}$ is a $J$-SSE of $H_{0}(\tau)$ by Theorem 5.5 . However, by Lemma 2.2, it can be verified that $H_{1}$ is not a SSE of $H_{0}(\tau)$.

\section{$6 \mathrm{~J}$-self-adjoint operator extensions of $H_{0}(\tau)$}

In this section, we discuss the characterization of all the $J$-self-adjoint operator extensions of $H_{0}(\tau)$ (i.e., $\left.H_{00}(\tau)\right)$.

It is evident that each $J$-self-adjoint operator extension (briefly, $J$-SOE) of $H_{0}(\tau)$ must be its $J$-SSE. So, the $J$-SSEs of $H_{0}(\tau)$ characterized in Section 5 contain all the $J$-SOEs of $H_{0}(\tau)$. With similar arguments to [23, Section 6], we can get the results for three different cases that $a=-\infty$ and $b=+\infty, a$ is finite and $b=+\infty$, and both $a$ and $b$ are finite.

Theorem 6.1 In the case that $a=-\infty$ and $b=+\infty$, each J-SSE of $H_{0}(\tau)\left(\right.$ i.e., $\left.H_{00}(\tau)\right)$ in Theorems 5.1-5.4 is its J-SOE.

Theorem 6.2 In the case that $a$ is finite and $b=+\infty$, a J-SSE of $H_{0}(\tau)\left(\right.$ i.e., $\left.H_{00}(\tau)\right)$ in Theorems 5.5 and 5.6 is its J-SOE if and only if the matrix $M$ in Theorems 5.5 and 5.6 
satisfies

$$
M\left(\begin{array}{c}
1 \\
-p(a-1)
\end{array}\right) \neq 0 .
$$

Theorem 6.3 In the case that both $a$ and $b$ are finite, a J-SSE of $H_{0}(\tau)\left(\right.$ i.e., $\left.H_{00}(\tau)\right)$ in Theorem 5.7 is its J-SOE if and only if the matrices $M$ and $N$ in Theorem 5.7 satisfy

$$
\operatorname{rank}\left(M\left(\begin{array}{c}
1 \\
-p(a-1)
\end{array}\right), N\left(\begin{array}{c}
0 \\
-p(b)
\end{array}\right)\right)=2 .
$$

\section{Examples for $\mathrm{J}$-self-adjoint subspace extensions}

In this section, we give three examples for $J$-self-adjoint subspace extensions.

Let $T$ be a subspace in $X^{2}$. The set

$$
\begin{aligned}
\Gamma(T):= & \{\lambda \in \mathbf{C}: \text { there exists } c(\lambda)>0 \text { such that } \\
& \|f-\lambda x\| \geq c(\lambda)\|x\| \text { for all }(x, f) \in T\}
\end{aligned}
$$

is called to be the regularity field of $T$. First, we give a result for the regularity field of $\Gamma\left(H_{0}(\tau)\right)$.

Lemma 7.1 Assume that a is finite. If for some $\lambda_{0}$, (1.1) has two linearly independent solutions in $L_{w}^{2}(I)$, then $\lambda_{0} \in \Gamma\left(H_{0}(\tau)\right)$, and consequently $\Gamma\left(H_{0}(\tau)\right)=\mathbf{C}$.

Proof By Lemma 2.5, let $y_{1}$ and $y_{2}$ be two linearly independent solutions of (1.1) such that $\left(y_{1}, y_{2}\right)(t)=1$. For $z \in L_{w}^{2}(I)$, set

$$
R_{\lambda}(z)(t):=\sum_{j=a}^{t-1}\left(y_{1}(t) y_{2}(j)-y_{1}(j) y_{2}(t)\right) w(j) z(j), \quad t \in\{t\}_{t=a-1}^{b},
$$

where $\sum_{j=a}^{a-2}=\sum_{j=a}^{a-1}=0$. Then it holds that

$$
(\tau-\lambda w(t)) R_{\lambda}(z)(t)=w(t) z(t), \quad t \in I .
$$

Further, it can be concluded that

$$
\left\|R_{\lambda}(z)\right\| \leq 2\left\|y_{1}\right\|\left\|y_{2}\right\|\|z\| .
$$

So, $R_{\lambda}$ is a bounded operator from $L_{w}^{2}(I)$ into $D(H(\tau))$. In addition, $\left(H_{00}(\tau)-\lambda\right)^{-1}$ is an operator in $L_{w}^{2}(I)$. Let $x \in D\left(H_{00}(\tau)\right)$ and take $z=\frac{1}{w}(\tau-\lambda w) x$. Then $(\tau-\lambda w)\left(x-R_{\lambda}(z)\right)=0$, i.e., $x-R_{\lambda}(z)$ is a solution of $(\tau-\lambda w) y=0$. Since

$$
x(a-1)-R_{\lambda}(z)(a-1)=x(a)-R_{\lambda}(z)(a)=0,
$$

one has that $x \equiv R_{\lambda}(z)$ on $I$. This yields that the operator $\left(H_{00}(\tau)-\lambda\right)^{-1}$ is a restriction of $R_{\lambda}$. Then $\left(H_{00}(\tau)-\lambda\right)^{-1}$ is a bounded operator and hence $\lambda \in \Gamma\left(H_{00}(\tau)\right)$. So, $\lambda \in \Gamma\left(H_{0}(\tau)\right)$ by $\Gamma\left(H_{0}(\tau)\right)=\Gamma\left(H_{00}(\tau)\right)$ and hence $\Gamma\left(H_{0}(\tau)\right)=\mathbf{C}$ by Lemma 3.7. This completes the proof. 
If $a$ is finite, then $d=1$ or 2 by Lemma 3.6. Further, by Lemma 7.1 the following result can be proved.

Theorem 7.1 Assume that a is finite. Then $d=2$ if and only if there are two linearly independent solutions of (1.1) in $L_{w}^{2}(I)$ and consequently $d=1$ if and only if there is at most one linearly independent solution of $(1.1)$ in $L_{w}^{2}(I)$.

Proof Let $d=2$. It can be verified by Lemmas 3.5 and 3.7 that (1.1) has two linearly independent solutions in $L_{w}^{2}(I)$. Conversely, suppose that there are two linearly independent solutions of $(1.1)$ in $L_{w}^{2}(I)$. Then $\Gamma\left(H_{0}(\tau)\right) \neq \emptyset$ by Lemma 7.1, and then by [26, Theorem $3.8], d=2$. This completes the proof.

Remark 7.1 In [17], Brown et al. developed a spectral theory for second-order differential operators with complex coefficients and one regular endpoint. They classified the corresponding formally second-order differential expressions into three limit cases at the singular endpoint: Cases I, II, and III. In [32], (1.1) was analogously classified into three limit cases at $b$ : Cases I, II, and III. By Lemma 7.1, $d_{b}=1$ if and only if (1.1) is in the limit Case I at $b$. Hence, (1.1) is not in the limit Case I at $b$ if and only if $d_{b}=2$. Further, $d=d_{b}$ by Theorem 4.1 if $a$ is finite.

Finally, we give three examples.

Example 7.1 Consider (1.1) on $I=\{t\}_{t=0}^{\infty}$ with $p(t)=w(t)=1$ and $q(t)=t^{2}$. It is noted that (1.1) is both $J$-symmetric and symmetric in this case. By [32, Corollary 3.2], equation (1.1) is in the limit Case I at $t=+\infty$. So, $d=1$ by Theorem 7.1. By Theorem 5.5, it can be concluded that (1.1) with the boundary condition

$$
m_{1} x(-1)+m_{2} \Delta x(-1)=0, \quad\left(m_{1}, m_{2}\right) \neq 0,
$$

determines all the $J$-SSEs of $H_{0}(\tau)$. In addition, (1.1) with the boundary condition

$$
\cos \alpha x(-1)+\sin \alpha \Delta x(-1)=0, \quad \alpha \in(0, \pi]
$$

determines all the $J$-SSEs of $H_{0}(\tau)$ which are also SSEs of $H_{0}(\tau)$. Especially, (7.3) contains the Dirichlet condition $x(-1)=0$ and the Neumann condition $\Delta x(-1)=0$.

Example 7.2 Consider (1.1) on $I=\{t\}_{t=0}^{\infty}$ with $p(t)=w(t)=1$ and $q(t)=t^{2}+i q_{2}(t)$, where $q_{2}$ is real-valued. By [32, Corollary 3.2], equation (1.1) is in the limit Case I at $t=+\infty$. So, $d=1$ by Theorem 7.1. By Theorem 5.5, it can be concluded that (1.1) with the boundary condition (7.3) determines all the $J$-SSEs of $H_{0}(\tau)$. Also, the condition $x(-1)=0$ and the condition $\Delta x(-1)=0$ are called the Dirichlet and Neumann boundary conditions, respectively.

Example 7.3 Consider (1.1) on $I=\{t\}_{t=0}^{\infty}$ with $p(t)=(t+1)^{4}, q(t)=-\mu$, where $\mu$ is a constant in the open upper half-plane and $w(t)=(t+1)^{-2}$. By [32, Example 3.2], equation (1.1) is not in the limit Case I at $t=+\infty$. So, $d=2$ by Theorem 7.1. Let $\phi_{1}$ and $\phi_{2}$ be solutions 
of (1.1) satisfying (5.15). By Theorem 5.6, (1.1) with the boundary conditions

$$
i x(-1)+\left(x, \phi_{2}\right)(+\infty)=0, \quad i p(-1) \Delta x(-1)+\left(x, \phi_{1}\right)(+\infty)=0
$$

determines a $J$-SSE of $H_{0}(\tau)$. In addition, (1.1) with the boundary conditions

$$
\begin{aligned}
& a x(-1)+b p(-1) \Delta x(-1)=0, \\
& c\left(x, \phi_{1}\right)(+\infty)+d\left(x, \phi_{2}\right)(+\infty)=0, \quad(a, b) \neq 0,(c, d) \neq 0,
\end{aligned}
$$

determines the $J$-SSEs of $H_{0}(\tau)$ with separated boundary conditions.

Remark 7.2 By Theorem 6.2, all the J-SSEs determined in terms of the Dirichlet or Neumann boundary conditions in Examples 7.1 and 7.2 are $J$-SOEs of $H_{0}(\tau)$.

\section{Competing interests}

The authors declare that they have no competing interests.

\section{Authors' contributions}

HS carried out the results of this article and drafted the manuscript. GR inspected the manuscript. All the authors read and approved the final manuscript.

\section{Author details}

${ }^{1}$ Department of Mathematics, Shandong University at Weihai, Weihai, Shandong 264209, P.R. China. ${ }^{2}$ School of Statistics and Mathematics, Shandong Economic University, Jinan, Shandong 250014, P.R. China.

\section{Acknowledgements}

The authors express their appreciation to the referees for their useful suggestions. This research was supported by the NNSF of China (Grant 11101241), the NNSF of Shandong Province (Grant ZR2011AQ002), the independent innovation fund of Shandong University (Grant 2011ZRYQ003), and the NNSFs of China (Grants 11071143 and 11226160).

Received: 30 January 2012 Accepted: 16 December 2012 Published: 7 January 2013

\section{References}

1. Glazman, IM: An analogue of the extension theory of Hermitian operators and a non-symmetric one-dimensional boundary-value problem on a half-axis. Dokl. Akad. Nauk SSSR 115, 214-216 (1957)

2. Qi, J, Zheng, Z, Sun, H: Classification of Sturm-Liouville equations with complex coefficients and operator realizations. Proc. R. Soc. Lond., Ser. A, Math. Phys. Eng. Sci. 467, 1835-1850 (2011)

3. Race, D: The theory of J-self-adjoint extensions of J-symmetric operators. J. Differ. Equ. 57, 258-274 (1985)

4. Shang, Z: On J-self-adjoint extensions of J-symmetric ordinary differential operators. J. Differ. Equ. 73, 153-177 (1988)

5. Garcia, SR, Putinar, M: Complex symmetric operators and applications. Trans. Am. Math. Soc. 358, 1285-1315 (2006)

6. Garcia, SR, Putinar, M: Complex symmetric operators and applications II. Trans. Am. Math. Soc. 359, 3913-3931 (2007)

7. Sun, H, Shi, Y: Self-adjoint extensions for linear Hamiltonian systems with two singular endpoints. J. Funct. Anal. 259, 2003-2027 (2010)

8. Weidmann, J: Spectral Theory of Ordinary Differential Operators. Lecture Notes in Mathematics, vol. 1258. Springer, Berlin (1987)

9. Weidmann, J: Linear Operators in Hilbert Spaces. Springer, New York (1980)

10. Everitt, WN, Markus, L: Boundary Value Problems and Symplectic Algebra for Ordinary Differential and Quasi-differential Operators. Math. Surveys Monogr., vol. 61. Am. Math. Soc., Providence (1999)

11. Knowles, IW: On the boundary conditions characterizing J-self-adjoint extensions of J-symmetric operators. J. Differ. Equ. 40, 263-289 (1981)

12. Sun, J: On the self-adjoint extensions of symmetric ordinary differential operators with middle deficiency indices. Acta Math. Sin. 2, 152-167 (1986)

13. Wang, A, Sun, J, Zettl, A: Characterization of domains of self-adjoint ordinary differential operators. J. Differ. Equ. 246 , 1600-1622 (2009)

14. Atkinson, FV: Discrete and Continuous Boundary Problems. Academic Press, New York (1964)

15. Behncke, H: Spectral theory of higher order differential operators. Proc. Lond. Math. Soc. 92, 139-160 (2006)

16. Behncke, H, Hinton, DB: Eigenfunctions, deficiency indices and spectra of odd order differential operators. Proc. Lond. Math. Soc. 97(2), 425-449 (2008)

17. Brown, BM, McCormack, DKR, Evans, WD, Plum, M: On the spectrum of second order differential operators with complex coefficients. Proc. R. Soc. Lond. A 455, 1235-1257 (1999)

18. Brown, BM, Marletta, M: Spectral inclusion and spectral exactness for singular non-self-adjoint Sturm-Liouville problems. Proc. R. Soc. Lond. A 457, 117-139 (2001) 
19. Edmunds, DE, Evans, WD: Spectral Theory and Differential Operators. Clarendon Press, Oxford (1987)

20. Lesch, M, Malamud, M: On the deficiency indices and self-adjointness of symmetric Hamiltonian systems. J. Differ Equ. 189, 556-615 (2003)

21. Sims, AR: Secondary conditions for linear differential operators of the second order. J. Math. Mech. 6, $247-285$ (1957)

22. Sun, H, Qi, J: On classification of Sturm-Liouville equations with complex coefficients. J. Math. Anal. Appl. 372 , 585-597 (2010)

23. Shi, Y, Sun, H: Self-adjoint extensions for second-order symmetric linear difference equations. Linear Algebra Appl. 434, 903-930 (2011)

24. Coddington, EA: Extension Theory of Formally Normal and Symmetric Subspaces. Mem. Amer. Math. Soc., 134 (1973)

25. Shi, Y: The Glazman-Krein-Naimark theory for Hermitian subspaces. J. Oper. Theory 68, 241-256 (2012)

26. Sun, H, Qi, J: The theory for J-Hermitian subspaces in a product space. ISRN Math. Anal. 2012, 1-16 (2012)

27. Shi, Y: Weyl-Titchmarsh theory for a class of discrete linear Hamiltonian systems. Linear Algebra Appl. 416, $452-519$ (2006)

28. Stević, S: Growth theorems for homogeneous second-order difference equations. ANZIAM J. 43, $559-566$ (2002)

29. Stević, S: Asymptotic behaviour of second-order difference equations. ANZIAM J. 46(1), 157-170 (2004)

30. Stević, S: Growth estimates for solutions of nonlinear second-order difference equations. ANZIAM J. 46(3), 439-448 (2005)

31. Sun, H, Qi, J, Jing, H: Classification of non-self-adjoint singular Sturm-Liouville difference equations. Appl. Math. Comput. 217, 8020-8030 (2011)

32. Sun, H, Qi, J: Criteria of the three cases for non-self-adjoint singular Sturm-Liouville difference equations. J. Differ. Equ. Appl. 18, 2069-2087 (2012)

33. Wilson, RH: Non-self-adjoint difference operators and their spectrum. Proc. R. Soc. Lond., Ser. A, Math. Phys. Eng. Sci. 461, 1505-1531 (2005)

doi:10.1186/1687-1847-2013-3

Cite this article as: Sun and Ren: J-self-adjoint extensions for second-order linear difference equations with complex coefficients. Advances in Difference Equations 2013 2013:3.

\section{Submit your manuscript to a SpringerOpen ${ }^{\ominus}$ journal and benefit from:}

- Convenient online submission

- Rigorous peer review

- Immediate publication on acceptance

- Open access: articles freely available online

- High visibility within the field

- Retaining the copyright to your article 